\title{
A multivariate approach in measuring innovation performance*
}

\author{
Elżbieta Roszko-Wójtowicz ${ }^{1}$, Jacek Białek ${ }^{2}$
}

\begin{abstract}
The goal of this research is to propose a procedure of innovativeness measurement, taking Summary Innovation Index methodology as a starting point. In contemporary world, innovative activity is perceived as a source of competitiveness and economic growth. New products, utility models, trademarks and creative projects are an important element of present socio-economic reality. In particular, authors focus on selection and application of multivariable statistical analysis to distinguish factors influencing innovativeness of EU economies to the highest degree. The result of quantitative analyses is linear ordering of EU countries by the level of their innovativeness based on the reduced set of diagnostic variables. The rating was compared with the outcome presented in Innovation Union Scoreboard (IUS) with Summary Innovation Index (SII). Conducted analysis proves a convergence between authors' results and existing ratings of innovativeness. Nevertheless, the main conclusion is that the methodology of innovativeness assessment remains an open issue and requires further research. Especially, it should first and foremost concentrate on deeper verification of a small set of variables that have the strongest impact on innovativeness. It is both, in economic and social interest, to get a clear picture of innovativeness driving forces.
\end{abstract}

Key words: innovativeness, Innovation Union Scoreboard, European Union, cluster analysis, factor analysis

JEL classification: $C 380,0300$

\footnotetext{
* Received: 01-09-2016; accepted: 09-12-2016

1 Assistant professor, Department of Economic and Social Statistics, Faculty of Economics and Sociology, University of Lodz, Rewolucji 1905 r. No. 41, 90-214 Łódź, Poland. Scientific affiliation: family entrepreneurship/businesses, lifelong learning, innovativeness, public procurement, statistics in economic and social research. Phone: +48 42635 4831. E-mail: eroszko33@gmail. com. Personal website: http://www.kseis.uni.lodz.pl/pracownicy/pracownik/id/13.

2 PhD with "habilitation", Assistant professor, Department of Statistical Methods, Faculty of Economics and Sociology, University of Lodz, Rewolucji 1905 r. No. 41/43, 90-214 Eódź, Poland. Scientific affiliation: mathematical statistics, price index theory, open pension funds efficiency.Phone: +4842635 5182.E-mail: jbialek@uni.lodz.pl.Personal website: http:// www.statystyka.uni.lodz.pl/pracownicy/dr-hab-inz-jacek-bialek/.
} 


\section{Introduction}

Innovativeness is an important constituent of economic competitiveness, it is part of constant and sustainable economic development. The abovementioned conditions increased interest in the problem of innovativeness, not only in its theoretical, but also practical aspects. In the age of globalisation, implementation and commercialisation of new technologies present the key element determining competitiveness of particular countries, therefore, the growth of innovativeness is perceived as a predominant direction of European Union society's transformation into information society. European countries' innovativeness has been assessed by European Commission on regular basis and consolidated are published in European Innovation Scoreboards (EIS). The history of research into innovativeness measures goes as far as the 1960s and originates from research and development investigation. In June 1963, in the Italian town of Frascati, the first version of Proposed Standard Practice for Surveys of Research and Development, also known as Frascati Manual, was released (W. Janasz (ed.), 2009: 129). There have been six versions of the manual so far. Since the fifth edition published in 1994, the main interest has shifted to research and development $(R \& D)$ as well as innovativeness which is the key element of knowledge economy. The last edition of 2002 is focused on R\&D statistics in the service sector and R\&D human resources.

Over 40 years of experience in working out the measures has resulted in a series of methodology manuals called Frascati Family. The most important manual from the Frascati family in the field of statistical research into innovative activity is Oslo Manual - Proposed guidelines for collecting and interpreting technological innovation data. Its first, 1992 edition, was prepared by OECD and the Nordic Industrial Fund, the second edition from 1997 was a result of OECD and Eurostat co-operation. Currently, the third version of the manual is n force. It's methodology, known as "Oslo methodology", sets international standards for measuring innovativeness. The latest version of Oslo manual contains extended typology, apart from product and process innovations, non-technological innovations have been added (i.e. organisational and marketing) and are treated equally as the aforementioned. Goldsmith and Foxall (2003) claim that measurement of innovativeness is highly dependent on the scientific goals of the researcher and the conception of innovativeness he or she adopts.

Research on innovativeness has its background, however, it constantly arouses lots of controversies and disagreement. A lively discussion in the field encouraged the authors to attempt at expressing their own view on a multi-dimensional assessment of innovativeness on the basis of partial indicators included in Summary Innovation Index. It must be added that research into countries' innovativeness is a complex issue. The year 2010 brought significant changes in measuring innovativeness on the European level. European Innovation Scoreboard (EIS) has been revised 
following the adoption of the Innovation Union Communication (European Commission, 2010). Basing on one decade of experience and critical opinions of previous methodology, a set of modified innovativeness indices in a form of Innovation Union Scoreboard (IUS) was implemented. EIS attitude was criticised for, among others, for: (Hollanders and Van Cruysen, 2008), quoted after (Piech, 2009):

- methodology of SII lacks theoretical background - instead of basing it on an innovation model, too strong emphasis is put on statistical aspects describing innovativeness,

- too much concentration on new technologies, though innovations take place no matter intensity of research and development work,

- co-linearity - certain indices are correlated, making the whole methodology $\mathrm{R} \& \mathrm{D}$ works oriented.

- problems with determining stimulants (not always the growth of a particular index value is beneficiary for the total innovativeness),

- there are usually difficulties with availability and completeness of data (data comes from different time horizons).

In this paper we limit ourselves to critical arguments with statistical nature. In particular we intend to avoid co-linearity of diagnostic variables and we use only normalized (by standardization) variables. Nevertheless, it is an important issue that measurement of innovativeness requires strong theoretical bases. Too much of an emphasis on research and development activities and at the same time neglecting broader context of innovation cannot only be regarded as a critical argument of SII methodology. It has to be stated that literature does not provide a clear evidence of an impact of R\&D public policy on private sector innovative activities and the general conclusion is still ambiguous (Radicic et al., 2014; David et al., 2000). For this reason $R \& D$ orientation in innovativeness measurement may distort the assessment of economic reality of a country.

Applying a modified Innovation Union Scoreboard (IUS) was aimed at monitoring implementation of Europe 2020 Innovation Union (European Commission, 2010) flagship by contrasting innovation performance of the EU27 Member States with relative strengths and weaknesses of their research and innovation systems. For the purpose of using IUS as a monitoring tool of the Innovation Union, the list of indicators used in the EIS 2009 had to be adapted.

A new list of 25 indicators replaced the former 29 indicators in IUS 2009 since they successfully illustrate the performance of national research and innovation systems considered as a whole. 19 of 29 indicators remained from last year's edition, 12 of which have not been changed, 2 indicators have been merged, and 5 indicators 
have been partly changed by using broadening or narrowing definitions or different denominators. Taking into account the merging of 2 indicators, 18 indicators IUS 2010 are equivalent to those of EIS 2009 and in addition 7 new indicators have been introduced (see Appendix A). In the literature it is a very common approach to group indicators into sub-systems or sub-indexes in measuring innovation capability (Saixing et al., 2010; Yuan-Chieh et al., 2012). Moreover, innovativeness is usually considered as a multi-dimensional and a complex phenomenon. The result of this can be seen in creation of innovativeness synthetic measures. These are mainly based on a huge number of variables from different economic and social areas (Matínez-Pellitero et al., 2008).

The research aims at working out a synthetic measure assessing EU member states innovativeness. A starting point for the analysis is a selection of data. Authors base their own approach to measurement of innovativeness on Summer Innovation Index methodology. The authors take a stand that current multivariate assessment of innovativeness is built on too many variables that are represented by one synthetic measure. It is important to propose a procedure enabling identification of the strongest innovation drivers. For this reason, authors concentrate on reducing the primary set of diagnostic variables and simultaneously distinguishing variables which best describe innovativeness of particular member states. The goal shall be achieved by application of various yet complementary methods of multidimensional statistics. The result of conducted statistical analysis is linear ordering of EU countries according to their innovation level based on a proposed synthetic measure. The following ranking will be compared with rating based on the Summary Innovation Index.

Hypothesis of the research later on in the paper verified and analyzed states that application of selected and mutually complementary methods of multidimensional statistics allows to obtain a set of diagnostic variables reduced to the smallest number, which is considered the base of developing a rating to assess EU member states innovativeness, whose results are convergent with those being presented in Innovation Union Scoreboard.

The paper is organized in the following manner. Introduction, where controversies around innovativeness measurement are presented, is followed by section 2, which provides readers with the evolution of innovation concept and the meaning of innovativeness for economic changes. These changes in understanding innovation are of a crucial importance for undertaking the further discussion on how to measure this multivariate phenomenon. Section 3 describes the methodology and conception used for analysis. Section 4 contains research data and empirical results of the analysis. Section 5 provides the results with their economic significance. Concluding remarks and suggestions for directions of future research and recommendations for innovativeness measurement are provided in Section 6. 


\section{Literature review}

So, what is innovation? Where does the phenomenon of constantly growing interest in the field of innovativeness come from?

The word "innovation" (lat. innovatis) stands for creating something completely new or renovating or improving the existing solutions. It can be implementation of something new, a newly introduced product, novelty or reform. Colloquially speaking, innovation is creating something new, which leads to a change for better (Janasz and Kozioł, 2007). As Porter (2001) stated innovation is something more than just scientific discovery. It "stretches beyond science and technology, and includes all the activities involving the discerning of needs and the transformation of knowledge into commercial products, processes, and services" (Porter, 2001: 6-7).

The milestone of economic thought on innovation starts with Schumpeterian theory (Schumpeter, 1946; Schumpeter, 1960). It is followed by the neoclassical growth model, as well known in literature as the Solow-Swan or exogenous growth model (Solow, 1956; Swan, 1956; Solow, 1957). The shortcomings of the neoclassical growth model encouraged economists to approach the issue of economic growth from a different perspective. In particular, the limitation of neoclassical growth model is that it cannot theoretically explain the essential driving-force of economic growth, the growth which is accelerated by an exogenous expansion of the knowledge stock (Uppenberg, 2009). The response to this comes with the endogenous growth model called the "AK-models". Modern endogenous growth models of the spillover type are explained in pioneering work of Harrod (1939) and Domar (1946). However, the most important modern AK-model, regarded as the starting point of modern endogenous growth theory, is that by Romer (1986). Significant contribution to the discussion on how innovation impacts growth has been demonstrated by Coe and Helpman (1995). Based on empirical studies, their attention is drawn to the fact that the more the country is open to trade the larger the positive impact of the foreign R\&D stock on total-factor productivity (TFP). The emphasis on this selection is to highlight the rationale for innovation which is the link of innovation and growth. Nevertheless, here the review concentrates on the evolution of innovation in the field of economics.

The term innovation was introduced into economic literature as early as in 1911, by an Austrian scientist - J.A. Schumpeter. At the root of Schumpeter's definition of innovativeness lies the answer to the question about the sources of income in capitalist economy. The answer corresponded to the times of the Great Depression, which happened in the years 1929-1933. The new approach highlighted the predominant role of enterprises in generating growth (Bielski, 2000). Schumpeter claimed that company's income results from introducing technical and organisational innovations to the market - new or cheaper production 
modes or means of producing completely new goods (Heilbroner, 1993). Accordingly, innovation process was described by Schumpeter as a creative activity incorporating creating, designing and implementing innovation. According to Schumpeter, in terms of activity, innovation stands for: (1) implementing a new product that customers had not dealt with before or a new type of a certain product; (2) implementing a new production mode that has not been exercised in a particular branch of industry; (3) opening a new market, being the one where a particular mode of domestic industry had existed before, no matter the market had existed or not; (4) achieving a new source of new or semi-finished products no matter the source had existed or it has just been created; (5) implementing new organisation of an industry, e.g. creating monopoly or breaking it up (Schumpeter, 1960).

The key element of this approach is the word "new". According to Schumpeter, the first application of a particular solution forms an integral part of the innovation definition (Niedzielski and Rychlik, 2006).

Intensified academic interest in innovativeness, however, came somewhat later, only in the 1940s/1950s. At the beginning, innovativeness was investigated on a macroeconomic scale; the influence of technical progress on economic growth was looked into. Microeconomic research was introduced later. Simultaneously, technological progress can be discussed in terms of the process and research allows for distinguishing its stages.

Definitions that were created after WW2 might be interpreted in a broad or narrow context. The narrow context relates innovation to technological aspects. It is connected with novelty that has been applied in practice. Providing fine characteristics of innovativeness essence seems to be of predominant importance. Therefore, Carter and Williams (1958: 29) defined innovativeness as "implementing an invention being part of unused technological knowledge". Meanwhile, Freeman (1994) as well as Janasz and Kozioł (2007) and Munsfield (1986) considered it as the first market appearance of a new product, process, system or device.

In a broader context, innovation is treated as a process comprising various activities, from the idea to realisation and implementation to the market. Innovation refers to creating, developing and introducing new values to products or new compilations of means and resources. The new solutions have to be new at least for the entity that introduces them (Niedzielski and Rychlik, 2006). Apart from Schumpeter, other flagship works comprise Herman, Hagen, Parker, Haffer, Allen, Rogers and Whitfield. The most genuine position on the list is the last one, according to which innovation concerns every modification that is based on assimilating the transferred knowledge (Whitfield, 1979).

In order to understand innovation better, it is important to take for granted, that it is never separated from other systems or actors present in the economy and 
society. Veblen (1971) claimed that institutional changes result from technological, social and economic changes. Social progress guaranteed by the institutions is inhibited by a necessity to modify existing procedures and adapting them to new circumstances. Veblen's theory of social evolution presents institutions as unable to catch up with all technological innovations, which may be reflected in a lower economic growth. Technology was equated with factors determining progress, i.e. quality, qualifications, and first and foremost, technical knowledge. He claimed progress to be dependent on modification of institutions which, in turn, could affect technological progress. North $(1981,2005)$, who developed several theories including the neoclassical theory of the state, claimed that ways of gaining knowledge as well as types of knowledge and skills are modified by institutions themselves, which has a specific influence on economic growth. Institutional change is affected by a constantly growing demand on education and qualifications as well as correlations between institutions and workers' knowledge potential. However, according to North, innovation does not present a significant factor for economic growth. The growth of economic wealth is conditioned by institutions formed in the process of the already mentioned social evolution understood as gaining knowledge and developing skills.

Porter (1990), an American economist known for his research into competitiveness, believed that innovation is a key word for economies' consolidation and increase of economic wealth. According to him, improvement of country's economies' competitiveness results from creating and implementing technological innovations. Factors that determine competitive advantage of a particular economy on the international market are: (1) access to production factors, (2) demand characteristics, (3) circumstances of company setting-up, (4) company management and activity, (4) interaction of familiar and supporting branches (Porter, 1990). Due to successful application of the factors, economy upgrades it competitiveness, and therefore, boosts its economic growth. Porter's model of a nation's economy development comprises four stages (1) factor-driven; (2) investment-driven; (3) innovation-driven; (4) wealthdriven. The third stage, i.e. innovation-driven, is crucial, since it stands for the boost of capacity to innovate, and entails economy's competitiveness and economic growth. The stage determines development that is driven by implemented innovations. It is them that improve and develop foreign ideas as well as create and initiate new technologies.

The key and pioneer role in interpreting the impact of innovation on economic growth was played by Fagerberg's research (1987). In his study, he discovered a catching-up effect. In countries with low GPD per capita, it may occur that economic growth rate will be higher than the forecasted index estimated on the basis of technological progress level and capital aggregation. The situation is determined by the fact that less advanced countries may benefit from the faster growth of wealthier countries by copying and importing their technologies. 
Technological progress, aggregation of knowledge, especially scientific and technical, as well as human capital are currently three main factors that influence economic growth. They determine contemporary economy more than production factors (land, labour and capital, knowledge that allows for efficient combination of other factors). Technological progress is a new source of competitiveness and has a considerable effect on stimulating economic growth, and in consequence - innovation.

\section{Methodology - Conception of analysis}

In this section a concept leading to identification of the strongest innovation drivers is being presented. Statistical procedure proposed in this paper was divided into several steps:

1. The first step was conducted using correlation analysis for the purpose of primary reduction of diagnostic variables, from the initial set of 25 variables. We tend to eliminate strongly correlated variables with relatively small volatility and thus we try to eliminate the co-linearity of explanatory variables.

2. In the second step, clustering of variables was done using Ward's hierarchical method with Euclidean distances. Generally, clustering is conducted for object class recognition, by searching most homogenous clusters (of closest possible distance within the cluster and maximum possible distance to other clusters). It may be also referred to as one of methods used for reduction of variables. In our case we use it just for reduction of diagnostic variables by obtaining the groups (clusters) of closely located variables. Clusters obtained through the lowest level of aggregation were later compared with covariance matrix identified a priori. Finally we eliminate variables having very small distance to at least one, more fluctuated variable.

3. Continuing, the third step concerned another reduction of diagnostic variables that was based on factor analysis carried out by means of Varimax rotation. Generally speaking, factor analysis is a statistical method used to describe variability among observed, correlated variables in terms of a potentially lower number of unobserved variables called factors. We identified six main factors and took for further analysis only variables with high factor loadings.

4. In the next step, clustering of EU countries was done using Ward's hierarchical method with Euclidean distance and $k$-means method. To compare the proposed methodology of innovativeness measurement to IUS methodology we considered two cases: clustering based on the full list of 25 variables and clustering based on the reduced list. In particular, we compared selected four clusters of countries obtained from $k$-means method $(k=4)$ to the classification of EU countries based on SII. 
5. The final next step was the ultimate EU countries rating regarding their innovativeness and innovation potential. The ranking was created by applying a non-pattern linear ordering with weighted and unweighted variant. In our opinion the weighted version is more intuitive because we assumed that the variable's weight is proportional to the identified variance multiplied by the factor from which the variable is derived. We compared EU member states' ratings created by means of linear ordering to SII rating. The convergence of both ratings was assessed with Spearman, Gamma and Kendall Tau correlation coefficients.

The analyses were carried out by means of Statistica 8.0 and MS Excel.

\section{Empirical data and analysis}

\subsection{Research data and descriptive statistics}

European Innovation Scoreboards presents a source of information on innovative activity in particular member states. European Innovation Scoreboards provide information on research and innovative activity outcome in Europe. The information allows particular countries and regions to create and implement initiatives corresponding to their needs. In 2000, within the Lisbon strategy, European Commission assigned using methodology based on innovation indicators measuring factors stimulating innovativeness level growth on the one hand, and factors evaluating effects of innovative actions taken by particular countries on the other hand. The obtained indicators' values, both single and summary, were published in yearly reports called European Innovation Scoreboard (EIS 2005 - EIS 2009). On the basis of European Innovation Scoreboard in 2010, a new tool was presented and first edition of the Innovation Union Scoreboard (IUS) was published. Over the years, both the number and the formula of innovation indicators applied have evolved. Independently of methodology changes in measuring, innovation scoreboards present a set of data contributing to assessing innovative efficiency of innovation actions taken during the investigated period, innovation level and innovative efficiency of particular EU member states. Thanks to aggregation of the indices, Summary Innovation Index was estimated and it was aimed at comparative analysis of innovation level in particular countries. They provide information on various areas relevant for the level. On the basis of SII indicator, innovation rating of analysed economies is created; countries are divided into four categories: innovation leaders, innovation followers, moderate innovators, modest innovators. At present SII, is estimated by means of 25 indices divided into five categories. The first three sets contain variables representing input level, and further two - output. Input is determined by a) factors stimulating innovativeness (EN. Enablers), which reflect conditions contributing to the development of innovativeness, that 
are not directly related to firm activity, b) firm activities - show innovative activity on the level of a firm. Output is described by business innovative activity results (European Commission, 2015).

Figure 1: Summary Innovation Index (SII) - structure

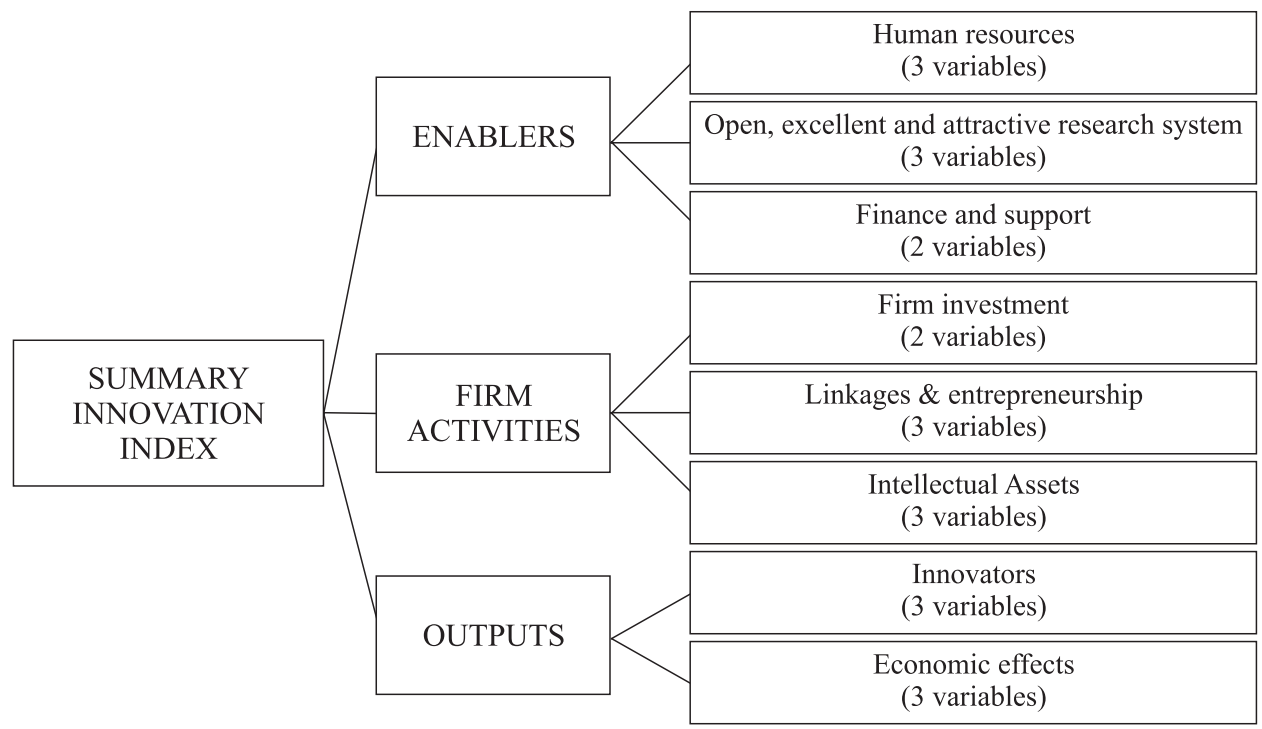

Source: Self-study based on European Commission, Innovation Union Scoreboard 2015, Internal Market Industry, Entrepreneurship and SMEs, European Union 2015, p. 7, http:// ec.europa.eu/growth/industry/innovation/facts-figures/scoreboards/index_en.htm Innovation Union

The estimated SII value provides basis for categorising European Union countries into four groups distinguished by different innovativeness levels of their economies.

The latest rating prepared for $28 \mathrm{EU}$ countries contains the following categorisation (European Commission, 2015):

Classification comprises four groups of countries (European Commission, 2015:

- The first group is made up by innovation leaders - the countries have SII index at least $20 \%$ higher than its average value for EU-28. Representatives: Sweden, Denmark, Germany, Finland;

- The second group are innovation followers - whose average SII in terms of innovativeness is less than $20 \%$ above and no more than $10 \%$ below average EU-28 value. Representatives: Holland, Luxembourg, Great Britain, Ireland, Belgium, France, Austria, Slovenia. 
- The third group - moderate innovators - their results are more than $10 \%$ and no less than $50 \%$ of the average. Representatives: Estonia, Czech Republic, Cyprus, Italy, Portugal, Malta, Spain, Hungary, Greece, Slovakia, Croatia, Poland, Lithuania);

- The last group - modest innovators - or catching-up countries - whose innovativeness results fall below 50\% average UE-28. Representatives: Latvia, Bulgaria, Romania.

Figure 2: Summary Innovation Index - comparison of EU member states' results 2014 vs. 2010

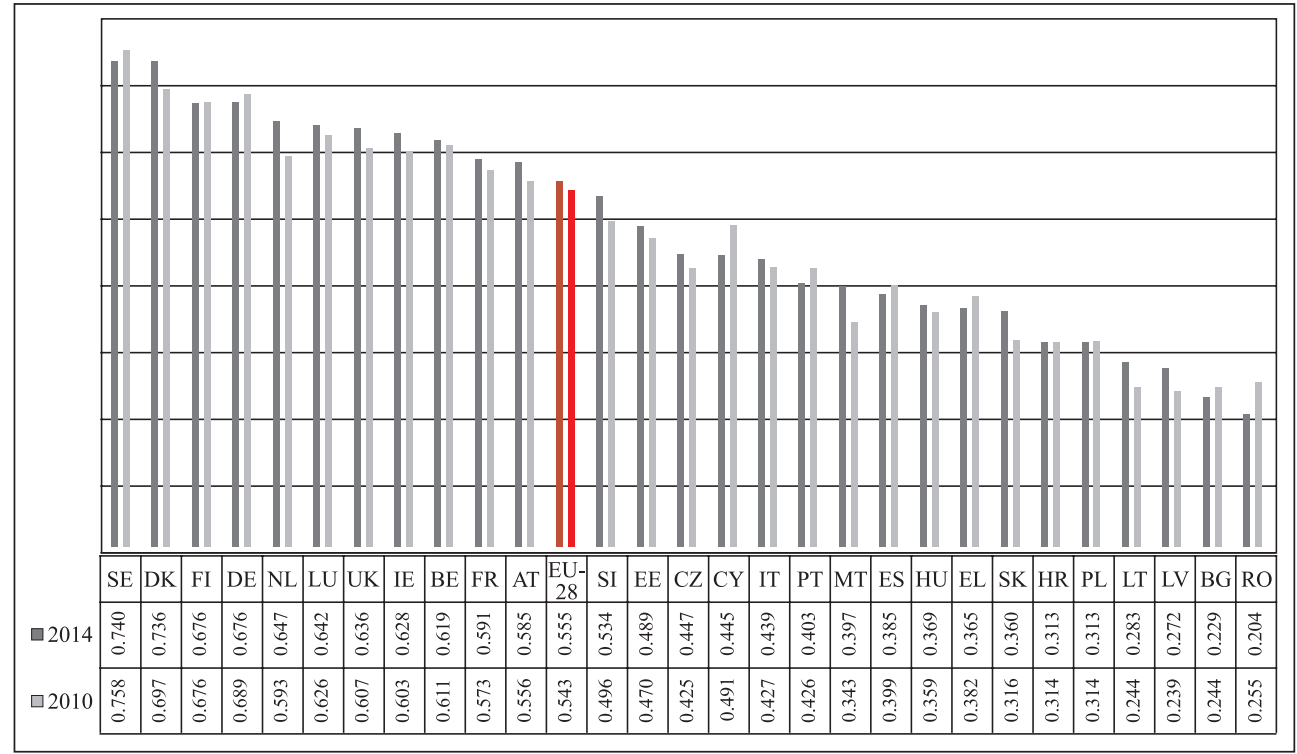

Source: Authors' self-study based on European Commission, Innovation Union Scoreboard 2015, Internal Market Industry, Entrepreneurship and SMEs, European Union 2015, Annex E, p. 92, http://ec.europa.eu/growth/industry/innovation/facts-figures/scoreboards/ index_en.htm

EIS is built from data coming from various primary research and sources as well as administrative data obtained from the European Patent Office and Domestic Market Harmonisation Office. Partial indices collected for EIS allow for creating Innovation Union Scoreboard based on a aggregate innovativeness index Summary Innovation Index SII - see Table 1. Further, the descriptive statistics measures of the considered variables are presented in Table 2. 
Table 1: SII - diagnostic indicators and single indicators values (based on IUS 2015)

\begin{tabular}{|c|c|c|c|c|c|}
\hline & $\begin{array}{c}\text { Innovativeness } \\
\text { measures }\end{array}$ & Poland & $\begin{array}{c}\text { EU-28 } \\
\text { average }\end{array}$ & Leader & Source \\
\hline \multicolumn{5}{|c|}{ 1. Human resources } & \\
\hline $\begin{array}{l}1.1 \\
{\left[\mathrm{X}_{1}\right]}\end{array}$ & $\begin{array}{l}\text { New doctorate graduates } \\
\text { (ISCED 6) per } 1000 \\
\text { population aged } 25-34\end{array}$ & 0.60 & 1.80 & $\begin{array}{l}\text { Sweden } \\
(2.80)\end{array}$ & Eurostat/Eurostat \\
\hline $\begin{array}{l}1.2 . \\
{\left[\mathrm{X}_{2}\right]}\end{array}$ & $\begin{array}{l}\text { Percentage population aged } \\
30-34 \text { having completed } \\
\text { tertiary education }\end{array}$ & 40.50 & 36.90 & $\begin{array}{l}\text { Ireland } \\
(52.60)\end{array}$ & Eurostat/Eurostat \\
\hline $\begin{array}{l}1.3 . \\
{\left[\mathrm{X}_{3}\right]}\end{array}$ & $\begin{array}{l}\text { Percentage youth aged } 20-24 \\
\text { having attained at least upper } \\
\text { secondary level education }\end{array}$ & 89.70 & 81.00 & $\begin{array}{l}\text { Croatia } \\
(95.0)\end{array}$ & Eurostat/Eurostat \\
\hline \multicolumn{5}{|c|}{ 2. Research systems } & \\
\hline $\begin{array}{l}2.1 \\
{\left[\mathrm{X}_{4}\right]}\end{array}$ & $\begin{array}{l}\text { International scientific } \\
\text { co-publications per million } \\
\text { population }\end{array}$ & 237.30 & 363.30 & $\begin{array}{l}\text { Denmark } \\
(1915.80)\end{array}$ & $\begin{array}{l}\text { Science-Metrix } \\
\text { using Scopus } \\
\text { data/Eurostat }\end{array}$ \\
\hline $\begin{array}{l}2.2 \\
{\left[\mathrm{X}_{5}\right]}\end{array}$ & $\begin{array}{l}\text { Scientific publications among } \\
\text { the top } 10 \% \text { most cited } \\
\text { publications worldwide as } \% \\
\text { of total scientific publications } \\
\text { of the country }\end{array}$ & 3.80 & 11.00 & $\begin{array}{l}\text { Netherlands } \\
\quad(15.60)\end{array}$ & $\begin{array}{l}\text { Science-Metrix } \\
\text { using Scopus } \\
\text { data/Science- } \\
\text { Metrix using } \\
\text { Scopus data }\end{array}$ \\
\hline $\begin{array}{l}2.3 . \\
{\left[\mathrm{X}_{6}\right]}\end{array}$ & $\begin{array}{l}\text { Non-EU doctorate students as } \\
\mathrm{a} \% \text { of all doctorate students }\end{array}$ & 1.90 & 25.50 & $\begin{array}{l}\text { France } \\
(35.40)\end{array}$ & Eurostat/Eurostat \\
\hline \multicolumn{5}{|c|}{ 3. Finance and suport } & \\
\hline $\begin{array}{l}3.1 \\
{\left[\mathrm{X}_{7}\right]}\end{array}$ & $\begin{array}{l}\text { Public R\&D expenditures as } \\
\% \text { of GDP }\end{array}$ & 0.48 & 0.72 & $\begin{array}{l}\text { Denmark } \\
(1.04)\end{array}$ & Eurostat/Eurostat \\
\hline $\begin{array}{l}3.2 . \\
{\left[\mathrm{X}_{8}\right]}\end{array}$ & $\begin{array}{l}\text { Venture capital (early stage, } \\
\text { expansion and replacement) } \\
\text { as \% of GDP }\end{array}$ & 0.036 & 0.062 & $\begin{array}{l}\text { Luxembourg } \\
\quad(0.188)\end{array}$ & Eurostat/Eurostat \\
\hline \multicolumn{5}{|c|}{ 4. Firm investments } & \\
\hline $\begin{array}{l}4.1 \\
{\left[\mathrm{X}_{9}\right]}\end{array}$ & $\begin{array}{l}\text { Business R\&D expenditures } \\
\text { as \% of GDP }\end{array}$ & 0.38 & 1.29 & $\begin{array}{l}\text { Finland } \\
(2.29)\end{array}$ & Eurostat/Eurostat \\
\hline $\begin{array}{c}4.2 . \\
{\left[X_{10}\right]}\end{array}$ & $\begin{array}{l}\text { Non-R\&D innovation } \\
\text { expenditures as } \% \text { of turnover }\end{array}$ & 1.04 & 0.69 & $\begin{array}{c}\text { Estonia } \\
(1.55)\end{array}$ & $\begin{array}{l}\text { Eurostat (CIS)/ } \\
\text { Eurostat (CIS) }\end{array}$ \\
\hline \multicolumn{5}{|c|}{ 5. Linkages \& entrepreneurship } & \\
\hline $\begin{array}{c}5.1 \\
{\left[\mathrm{X}_{11}\right]}\end{array}$ & $\begin{array}{l}\text { SMEs innovating in-house as } \\
\% \text { of SMEs }\end{array}$ & 10.10 & 28.70 & $\begin{array}{l}\text { Netherlands } \\
(38.90)\end{array}$ & $\begin{array}{l}\text { Eurostat/Eurostat } \\
\text { (CIS) }\end{array}$ \\
\hline $\begin{array}{c}5.2 \\
{\left[\mathrm{X}_{12}\right]}\end{array}$ & $\begin{array}{l}\text { Innovative SMEs collaborating } \\
\text { with others as \% of SMEs }\end{array}$ & 3.90 & 10.30 & $\begin{array}{c}\text { Belgium } \\
(22.90)\end{array}$ & $\begin{array}{l}\text { Eurostat (CIS)/ } \\
\text { Eurostat (CIS) }\end{array}$ \\
\hline $\begin{array}{l}5.3 . \\
{\left[\mathrm{X}_{13}\right]}\end{array}$ & $\begin{array}{l}\text { Public-private co-publications } \\
\text { per million population }\end{array}$ & 4.70 & 50.30 & $\begin{array}{l}\text { Denmark } \\
(193.00)\end{array}$ & $\begin{array}{l}\text { Centre for Science } \\
\text { and Technology } \\
\text { Studies (CWTS) } \\
\text { using Thomson } \\
\text { Reuters data/ } \\
\text { Eurostat }\end{array}$ \\
\hline
\end{tabular}


Elżbieta Roszko-Wójtowicz, Jacek Białek • A multivariate approach in measuring...

\begin{tabular}{|c|c|c|c|c|c|}
\hline & $\begin{array}{c}\text { Innovativeness } \\
\text { measures }\end{array}$ & Poland & \begin{tabular}{|c|} 
EU-28 \\
average \\
\end{tabular} & Leader & Source \\
\hline 6. Inte & lectual assets & & & & \\
\hline $\begin{array}{c}6.1 \\
{\left[\mathrm{X}_{14}\right]}\end{array}$ & $\begin{array}{l}\text { PCT patents applications per } \\
\text { billion GDP (in PPS } € \text { ) }\end{array}$ & 0.42 & 3.78 & $\begin{array}{c}\text { Finland } \\
(9.37) \\
\end{array}$ & OECD/Eurostat \\
\hline $\begin{array}{c}6.2 . \\
{\left[X_{15}\right]}\end{array}$ & $\begin{array}{l}\text { PCT patent applications in } \\
\text { societal challenges per billion } \\
\text { GDP (in PPS } € \text { ) (climate } \\
\text { change mitigation; health) } \\
\end{array}$ & 0.09 & 0.98 & $\begin{array}{l}\text { Denmark } \\
(2.67)\end{array}$ & OECD/Eurostat \\
\hline $\begin{array}{c}6.3 . \\
{\left[X_{16}\right]}\end{array}$ & $\begin{array}{l}\text { Community trademarks per } \\
\text { billion GDP (in PPS } € \text { ) }\end{array}$ & 3.61 & 5.83 & $\begin{array}{l}\text { Malta } \\
(30.97)\end{array}$ & $\begin{array}{l}\text { Office for } \\
\text { Harmonization } \\
\text { in the Internal } \\
\text { Market/Eurostat } \\
\end{array}$ \\
\hline $\begin{array}{l}6.4 . \\
{\left[X_{17}\right]}\end{array}$ & $\begin{array}{l}\text { Community designs per } \\
\text { billion GDP (in PPS€) }\end{array}$ & 1.62 & 1.13 & $\begin{array}{l}\text { Luxembourg } \\
\qquad(2.44)\end{array}$ & $\begin{array}{l}\text { Office for } \\
\text { Harmonization } \\
\text { in the Internal } \\
\text { Market/Eurostat } \\
\end{array}$ \\
\hline 7. Inno & vators & & & & \\
\hline $\begin{array}{c}7.1 . \\
{\left[\mathrm{X}_{18}\right]}\end{array}$ & $\begin{array}{l}\text { SMEs introducing product or } \\
\text { process innovations as } \% \text { of } \\
\text { SMEs }\end{array}$ & 13.10 & 30.60 & $\begin{array}{c}\text { Luxembourg } \\
\quad(43.10)\end{array}$ & $\begin{array}{l}\text { Eurostat (CIS)/ } \\
\text { Eurostat (CIS) }\end{array}$ \\
\hline $\begin{array}{l}7.2 . \\
{\left[\mathrm{X}_{19}\right]}\end{array}$ & $\begin{array}{l}\text { SMEs introducing marketing } \\
\text { or organizational innovations } \\
\text { as } \% \text { of SMEs }\end{array}$ & 14.20 & 36.20 & $\begin{array}{l}\text { Luxembourg } \\
\quad(52.10)\end{array}$ & $\begin{array}{l}\text { Eurostat (CIS)/ } \\
\text { Eurostat (CIS) }\end{array}$ \\
\hline $\begin{array}{c}7.3 \\
{\left[\mathrm{X}_{20}\right]}\end{array}$ & $\begin{array}{l}\text { Employment in fast-growing } \\
\text { firms of innovative sectors }\end{array}$ & 19.30 & 17.90 & $\begin{array}{l}\text { Ireland } \\
(21.80) \\
\end{array}$ & Eurostat/Eurostat \\
\hline 8. Ecol & 1omic effects & & & & \\
\hline $\begin{array}{l}8.1 \\
{\left[\mathrm{X}_{21}\right]}\end{array}$ & $\begin{array}{l}\text { Employment in knowledge- } \\
\text { intensive activities } \\
\text { (manufacturing and services) } \\
\text { as \% of total employment } \\
\end{array}$ & 9.60 & 13.80 & $\begin{array}{l}\text { Luxembourg } \\
\quad(26.20)\end{array}$ & Eurostat/Eurostat \\
\hline $\begin{array}{l}8.2 . \\
{\left[\mathrm{X}_{22}\right]}\end{array}$ & $\begin{array}{l}\text { Medium and high-tech } \\
\text { product exports as } \% \text { of total } \\
\text { product exports }\end{array}$ & 48.60 & 53.00 & $\begin{array}{l}\text { Hungary } \\
(66.33)\end{array}$ & $\begin{array}{l}\text { Eurostat / United } \\
\text { Nations/ Eurostat } \\
\text { / United Nations }\end{array}$ \\
\hline $\begin{array}{l}8.3 \\
{\left[\mathrm{X}_{23}\right]}\end{array}$ & $\begin{array}{l}\text { Knowledge-intensive services } \\
\text { exports as } \% \text { total service } \\
\text { exports }\end{array}$ & 33.60 & 49.50 & $\begin{array}{l}\text { Ireland } \\
(76.10)\end{array}$ & Eurostat/Eurostat \\
\hline $\begin{array}{l}8.4 \\
{\left[\mathrm{X}_{24}\right]}\end{array}$ & $\begin{array}{l}\text { Sales of new to market and } \\
\text { new to firm innovations as \% } \\
\text { of turnover }\end{array}$ & 6.30 & 12.40 & $\begin{array}{l}\text { Denmark } \\
(22.10)\end{array}$ & $\begin{array}{l}\text { Eurostat (CIS)/ } \\
\text { Eurostat (CIS) }\end{array}$ \\
\hline $\begin{array}{c}8.5 \\
{\left[\mathrm{X}_{25}\right]}\end{array}$ & $\begin{array}{l}\text { License and patent revenues } \\
\text { from abroad as \% of GDP }\end{array}$ & 0.06 & 0.65 & $\begin{array}{c}\text { Netherlands } \\
(3.75)\end{array}$ & Eurostat/Eurostat \\
\hline
\end{tabular}

Note: SII reaches values from 0 (low innovativeness) to 1 (high innovativeness).

Source: Authors' self-study based on European Commission, Innovation Union Scoreboard 2015, Internal Market Industry, Entrepreneurship and SMEs, Belgium 2015, Annex A: Current performance, pp. 82-83, http://ec.europa.eu/growth/industry/innovation/factsfigures/scoreboards/index_en.htm 
On the initial step of the analysis, i.e. observing data from Table 2 , it can be noticed that the volatility of most of considered variables in on the acceptable level. In fact, although, authors put their attention to variables with rather low fluctuations (like $X_{3}$ or $X_{20}$ ), no assumption on a minimal requirements for the volatility level was drawn.

Table 2: Descriptive statistics for the considered variables

\begin{tabular}{|c|r|r|r|r|r|r|r|r|}
\hline Variable & \multicolumn{1}{|c|}{ Mean } & Median & \multicolumn{1}{c|}{ Min. } & \multicolumn{1}{|c|}{ Max. } & Variance & Std.Dev. & Coef.Var. & Skewness \\
\hline$X_{1}$ & 1.6286 & 1.7000 & 0.2000 & 2.800 & 0.6 & 0.7428 & 45.6103 & -0.235671 \\
\hline$X_{2}$ & 37.9250 & 40.6000 & 22.4000 & 52.600 & 91.9 & 9.5867 & 25.2780 & -0.148367 \\
\hline$X_{3}$ & 83.4607 & 85.8000 & 63.8000 & 95.000 & 53.6 & 7.3212 & 8.7721 & -0.911185 \\
\hline$X_{4}$ & 864.3567 & 764.3869 & 187.8981 & 1915.773 & 264770.1 & 514.5581 & 59.5308 & 0.498397 \\
\hline$X_{5}$ & 8.7079 & 9.5600 & 2.9600 & 15.570 & 15.0 & 3.8687 & 44.4276 & -0.044819 \\
\hline$X_{6}$ & 10.7899 & 7.0336 & 0.0696 & 35.399 & 102.9 & 10.1460 & 94.0324 & 0.895776 \\
\hline$X_{7}$ & 0.6175 & 0.5650 & 0.2500 & 1.040 & 0.1 & 0.2373 & 38.4231 & 0.376754 \\
\hline$X_{8}$ & 0.0545 & 0.0545 & 0.0005 & 0.188 & 0.0 & 0.0396 & 72.6443 & 1.374620 \\
\hline$X_{9}$ & 0.9696 & 0.7700 & 0.0700 & 2.290 & 0.5 & 0.6960 & 71.7814 & 0.580500 \\
\hline$X_{10}$ & 0.6771 & 0.5879 & 0.1412 & 1.555 & 0.1 & 0.3805 & 56.1996 & 0.758340 \\
\hline$X_{11}$ & 26.3537 & 27.6793 & 10.1252 & 38.940 & 102.3 & 10.1121 & 38.3706 & -0.366957 \\
\hline$X_{12}$ & 10.5265 & 11.5045 & 1.1999 & 22.879 & 31.9 & 5.6483 & 53.6582 & 0.399144 \\
\hline$X_{13}$ & 46.8242 & 28.9070 & 1.4531 & 192.988 & 2208.3 & 46.9928 & 100.3600 & 1.532564 \\
\hline$X_{14}$ & 2.6592 & 1.5887 & 0.2020 & 9.371 & 7.6 & 2.7505 & 103.4359 & 1.258818 \\
\hline$X_{15}$ & 0.6921 & 0.4446 & 0.0000 & 2.673 & 0.5 & 0.6860 & 99.1146 & 1.273729 \\
\hline$X_{16}$ & 7.4670 & 5.3235 & 1.2433 & 30.973 & 52.1 & 7.2212 & 96.7083 & 2.512673 \\
\hline$X_{17}$ & 1.1405 & 1.1054 & 0.1489 & 2.439 & 0.4 & 0.6327 & 55.4815 & 0.172962 \\
\hline$X_{18}$ & 29.0217 & 32.2004 & 5.1572 & 43.062 & 120.3 & 10.9679 & 37.7920 & -0.566928 \\
\hline$X_{19}$ & 34.7053 & 36.2795 & 14.1939 & 52.052 & 103.4 & 10.1705 & 29.3054 & -0.345769 \\
\hline$X_{20}$ & 17.0204 & 16.9549 & 11.3000 & 21.800 & 5.6 & 2.3722 & 13.9374 & -0.343828 \\
\hline$X_{21}$ & 13.8179 & 13.6000 & 6.5000 & 26.200 & 16.7 & 4.0873 & 29.5798 & 0.860808 \\
\hline$X_{22}$ & 46.7724 & 47.4105 & 17.9870 & 66.331 & 139.9 & 11.8288 & 25.2902 & -0.399699 \\
\hline$X_{23}$ & 40.0783 & 35.3757 & 14.2356 & 76.118 & 267.2 & 16.3456 & 40.7843 & 0.774954 \\
\hline$X_{24}$ & 10.6061 & 10.7642 & 3.6898 & 22.102 & 17.5 & 4.1812 & 39.4228 & 0.708242 \\
\hline$X_{25}$ & 0.5354 & 0.1594 & 0.0054 & 3.749 & 0.7 & 0.8318 & 155.3659 & 2.628984 \\
\hline
\end{tabular}

Note: The analyses were carried out by means of Statistica 8.0 and MS Excel.

Source: Authors' calculations based on European Commission, Innovation Union Scoreboard 2015, Internal Market Industry, Entrepreneurship and SMEs, Belgium 2015, Annex A: Current performance, pp. 82-83, http://ec.europa.eu/growth/industry/innovation/factsfigures/scoreboards/index_en.htm

The biggest values of the coefficients of variance were achieved in the case of the following variables: $X_{6}, X_{13}, X_{14}, X_{15}, X_{16}$ and $X_{25}$. However, in the final, reduced set of variables only two of them are included $\left(X_{13}\right.$ and $\left.X_{14}\right)$. Moreover, although the $X_{3}$ variable has the lowest coefficient of variance (only $8.7 \%$ ) it is characterized by 
quite high negative asymmetry and a big value of the factor loading connected with the identified fifth factor (see Table 3). Thus, authors decided to take this variable into account in building the innovativeness rating (see Sections 4.4. and 5).

\subsection{Preselection of data}

During the first stage, correlation analysis was applied to reduce the initial number of variables. From all pairs of variables where Pearson correlation coefficient was at least 0.9 , for further analysis we took the variable with a higher volatility coefficient based on standard deviation. This procedure allowed elimination of colinearity of explanatory variables, maintaining the most significant variables for the research at the same time. We identified the following pairs of strongly correlated variables: $X_{9}$ and $X_{14}$ (their correlation coefficient equals $\left.\rho_{9,14}=0.9\right), X_{11}$ and $X_{18}\left(\rho_{11,18}\right.$ $=0.97), X_{13}$ and $X_{15}\left(\rho_{13,15}=0.94\right), X_{14}$ and $X_{15}\left(\rho_{14,15}=0.94\right)$. According to the abovementioned criterion we rejected the following variables: $X_{9}, X_{15}$ and $X_{18}$. Thus, the total number of variables was reduced from 25 to 22 .

\subsection{Reducing the number of variables by means of cluster analysis}

Cluster analysis or clustering is the task of grouping a set of objects in such a way that objects in the same group (called a cluster) are more similar (in some sense or another) to each other than to those in other groups (clusters). Connectivity based clustering, also known as hierarchical clustering, is based on the core idea of objects being more related to nearby objects than to objects farther away. These algorithms connect "objects" to form "clusters" based on their distance. A cluster can be described largely by the maximum distance needed to connect parts of the cluster (Ken, 1994; Everitt et al., 2011). The next step towards dimensionality reduction of explanatory variables was clustering of variables describing objects (countries) $^{3}$. The analysis was supposed to distinguish variables creating clusters i.e. most similar variables (of the lowest value of Euclidean value).

For the analysis, Ward's agglomerative method (1963) with Euclidean distances was applied (Figure 3). Clusters obtained through the lowest level of aggregation were later compared with covariance matrix identified a priori. The Euclidean distances are the smallest in the case of the following cluster from the lowest level of aggregation: $X_{1}, X_{7}, X_{8}, X_{10}, X_{17}$ and $X_{25}$.

\footnotetext{
$\overline{3}$ Generally, clustering is conducted for object class recognition, but it may be referred to as one of methods used for reduction of variables.
} 
Figure 3: Tree diagram for 22 variables obtained by Ward's method

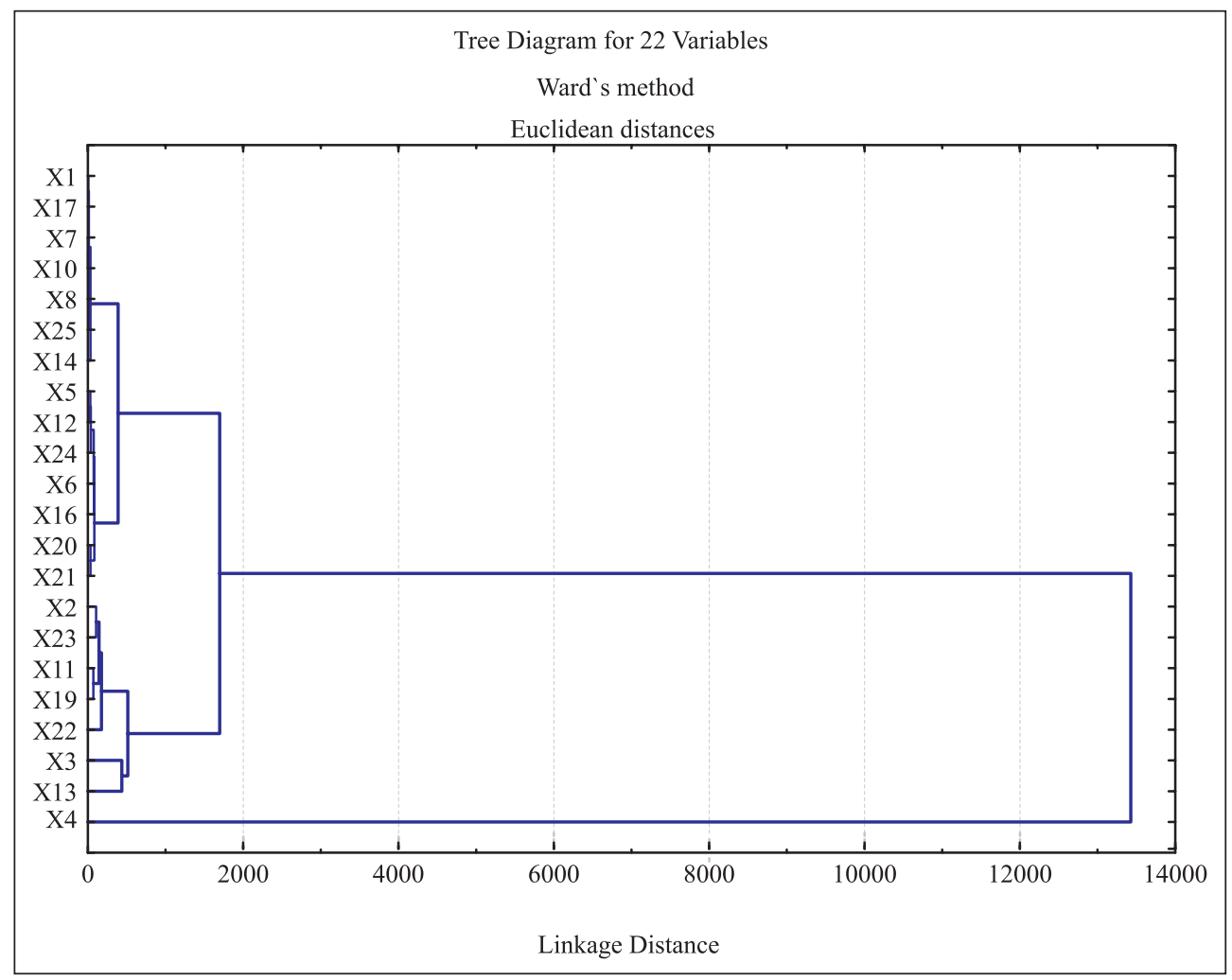

Note: The analyses were carried out by means of Statistica 8.0 and MS Excel.

Source: Authors' calculations based on European Commission, Innovation Union Scoreboard 2015, Internal Market Industry, Entrepreneurship and SMEs, Belgium 2015, Annex A: Current performance, pp. 82-83, http://ec.europa.eu/growth/industry/innovation/factsfigures/scoreboards/index_en.htm

Due to the assumed reduction criterion we eliminated variables with relatively small variation among variables in this selected cluster, namely we roll out: $X_{1}$ and $X_{7}$. In other words, we took only 20 variables for further analysis.

\subsection{Reducing the number of variables by factor analysis}

Factor analysis is a statistical method used to describe variability among observed, correlated variables in terms of a potentially lower number of unobserved variables called factors (Child, 2006; Thomson, 2004). The observed variables are modeled as linear combinations of the potential factors, plus "error" terms. Mathematically speaking, the object of factor analysis is matrix of data containing $n$ number of $m$ variables $X=\left[x_{i j}\right]_{n \times m}$, where $i=1,2, \ldots, n$, 
$j=1,2, \ldots, m$. As a result of transforming the value of variables by means of standardization formula we achieve variables of identical expected value (equals 0 ) and unit standard deviation: $Z=\left[z_{i j}\right]_{n \times m}$. It is assumed there is a relation between $X_{j}$ variables whose loading and direction is determined by Pearson's linear correlation coefficients included in matrix: $R=\left[r_{k j}\right]_{m \times m}$. In factor analysis, the basis for identifying common and unique components is classification of variances into common and unique, which together represent the total variance related to a specific variable. On making appropriate assumptions regarding common and unique factors, (Child, 2006; Walesiak, 1996), mathematical model of factor analysis may be written as matrix:

$$
Z=A F+B U
$$

where: $Z$ - variables matrix $A$ - matrix of factor loadings for common components, $F$-common factors matrix, $B$ - matrix of factor loadings for specific components, $U$ - unique factors matrix. In the presented model, the structure of interdependencies within the primary set of variables is represented by covariance matrix:

$$
V=A^{T}+B^{2}
$$

After removing from equation a component representing unique variance, one gets the so called reduced correlation matrix:

$$
\widetilde{R}=A A^{T}
$$

The basic objective of factor analysis is solving the equation (3) by matrix $A$, i.e. determining factor loadings for common components. Next, the reduced set of 20 variables underwent factor analysis. Many variables in the set were still highly correlated, which led to carrying out factor analysis. Principal components method was used to distinguish most relevant factors and corresponding factor loadings ${ }^{4}$ (Walesiak, 1996). Yet, Varimax rotation ${ }^{5}$ was introduced to maximize the variance of primeval factor loadings on variables. Selected factors and corresponding factor loadings established for particular variables are presented in Table 3.

\footnotetext{
4 The factor loadings, also called component loadings, are the correlation coefficients between the cases (rows) and factors (columns). The squared factor loading is the percent of variance in that indicator variable explained by the factor. By one rule of thumb in confirmatory factor analysis (CFA), loadings should be 0.7 or higher to confirm that independent variables identified a priori are represented by a particular factor.

5 Varimax rotation is an orthogonal rotation of the factor axes to maximize the variance of the squared loadings of a factor (column) on all the variables (rows) in a factor matrix, which has the effect of differentiating the original variables by extracted factor. Each factor will tend to have either large or small loadings of any particular variable. A varimax solution yields results which make it as easy as possible to identify each variable with a single factor.
} 
Table 3: Factors and corresponding factor loadings

\begin{tabular}{|c|r|r|r|r|r|r|}
\hline Variable & \multicolumn{1}{|c|}{ Factor - 1 } & \multicolumn{1}{|c|}{ Factor - 2 } & \multicolumn{1}{|c|}{ Factor - 3 } & \multicolumn{1}{|c|}{ Factor - 4 } & \multicolumn{1}{|c|}{ Factor - 5 } & \multicolumn{1}{|c|}{ Factor - 6 } \\
\hline$X_{2}$ & 0.318365 & 0.190899 & -0.209892 & $0.765057^{*}$ & -0.192167 & 0.067951 \\
\hline$X_{3}$ & -0.101233 & -0.200536 & 0.089851 & 0.044754 & $-0.905317^{*}$ & -0.098013 \\
\hline$X_{4}$ & $0.764142^{*}$ & 0.282649 & 0.053039 & 0.352136 & 0.081959 & 0.345782 \\
\hline$X_{5}$ & 0.681240 & 0.079636 & 0.022667 & 0.343928 & 0.338640 & 0.488307 \\
\hline$X_{6}$ & 0.484401 & -0.025545 & 0.207759 & 0.526404 & 0.372341 & 0.246644 \\
\hline$X_{8}$ & 0.304361 & 0.462494 & 0.048909 & 0.584708 & 0.028895 & 0.151318 \\
\hline$X_{10}$ & -0.232034 & 0.131197 & -0.268790 & -0.493870 & -0.496917 & 0.012116 \\
\hline$X_{11}$ & 0.548567 & 0.321109 & 0.047088 & 0.113272 & 0.090869 & 0.636727 \\
\hline$X_{12}$ & 0.617004 & -0.046339 & -0.034306 & 0.236638 & -0.227839 & 0.551182 \\
\hline$X_{13}$ & $0.914729^{*}$ & -0.017069 & 0.114761 & 0.124116 & 0.120832 & 0.157230 \\
\hline$X_{14}$ & $0.897735^{*}$ & 0.054102 & 0.145927 & 0.112346 & 0.021567 & 0.097140 \\
\hline$X_{16}$ & -0.095592 & $0.924953^{*}$ & 0.049043 & 0.077281 & 0.133362 & 0.177467 \\
\hline$X_{17}$ & 0.584804 & 0.687852 & 0.094073 & 0.006779 & 0.061334 & -0.021759 \\
\hline$X_{19}$ & 0.247619 & 0.355881 & 0.113656 & 0.117883 & 0.104570 & $0.803233^{*}$ \\
\hline$X_{20}$ & 0.087317 & 0.033990 & $0.852890^{*}$ & 0.302566 & -0.030579 & 0.211357 \\
\hline$X_{21}$ & 0.297049 & 0.600014 & 0.224962 & 0.507647 & 0.025726 & 0.411125 \\
\hline$X_{22}$ & 0.136853 & 0.168526 & $0.856815^{*}$ & -0.217017 & -0.025492 & -0.035392 \\
\hline$X_{23}$ & 0.089359 & 0.054813 & 0.205642 & 0.594878 & 0.118587 & 0.501658 \\
\hline$X_{24}$ & 0.282761 & -0.228844 & 0.373253 & -0.153552 & 0.292802 & 0.502068 \\
\hline$X_{25}$ & 0.494736 & 0.071526 & 0.148092 & 0.497006 & 0.117926 & 0.015693 \\
\hline
\end{tabular}

Note: $(*)$ Meaningful factor loadings ( $>0.7)$ are bolded in the table; The analyses were carried out by means of Statistica 8.0 and MS Excel.

Source: Authors' calculations based on European Commission, Innovation Union Scoreboard 2015, Internal Market Industry, Entrepreneurship and SMEs, Belgium 2015, Annex A: Current performance, pp. 82-83, http://ec.europa.eu/growth/industry/innovation/factsfigures/scoreboards/index_en.htm

Marked values in the table concern only these variables which are important from the point of view of further analysis. Eigenvalues of correlation matrix are represented in Table 4, which contains e.g. information on the percentage of the total variance identified by a specific factor. In addition, to confirm the number of assumed factors, the spree plot was applied (compare Figure 4). 
Table 4: Eigenvalues and percentage of variances explained by factors

\begin{tabular}{|c|r|r|r|r|}
\hline Factor No. & Eigenvalue & \multicolumn{1}{c|}{$\begin{array}{c}\text { \% Total }- \\
\text { variance }\end{array}$} & $\begin{array}{c}\text { Cumulative }- \\
\text { Eigenvalue }\end{array}$ & Cumulative $-\%$ \\
\hline 1 & 8.617813 & 43.08906 & 8.61781 & 43.08906 \\
\hline 2 & 1.919895 & 9.59947 & 10.53771 & 52.68854 \\
\hline 3 & 1.748885 & 8.74443 & 12.28659 & 61.43296 \\
\hline 4 & 1.380110 & 6.90055 & 13.66670 & 68.33351 \\
\hline 5 & 1.324517 & 6.62259 & 14.99122 & 74.95610 \\
\hline 6 & 1.106675 & 5.53338 & 16.09790 & 80.48948 \\
\hline
\end{tabular}

Note: The analyses were carried out by means of Statistica 8.0 and MS Excel.

Source: Authors' calculations based on European Commission, Innovation Union Scoreboard 2015, Internal Market Industry, Entrepreneurship and SMEs, Belgium 2015, Annex A: Current performance, pp. 82-83, http://ec.europa.eu/growth/industry/innovation/factsfigures/scoreboards/index_en.htm

According to Kaiser's criterion (1960), only factors with eigenvalues greater than 1 should be retained for further analysis. In this case, there are 6 factors of this kind (see Table 4), altogether they identify more than $80 \%$ total variance of variables, which is acceptable. Catell's spree plot (1966) appears to confirm the number of selected factors, since the spree begins around the fifth factor (compare Figure 4).

It is worth noticing that many of variables retained for the analysis do not have loadings significant in terms of the six factors. Following the assumed procedure, variables are removed from further analysis. Therefore, the factor analysis resulted in considering only the following variables: $X_{2}, X_{3}, X_{4}, X_{13}, X_{14}, X_{16}, X_{19}, X_{20}$ and $X_{22}$ (9 variables are taken for further analysis). 
Figure 4: Plot of eigenvalues

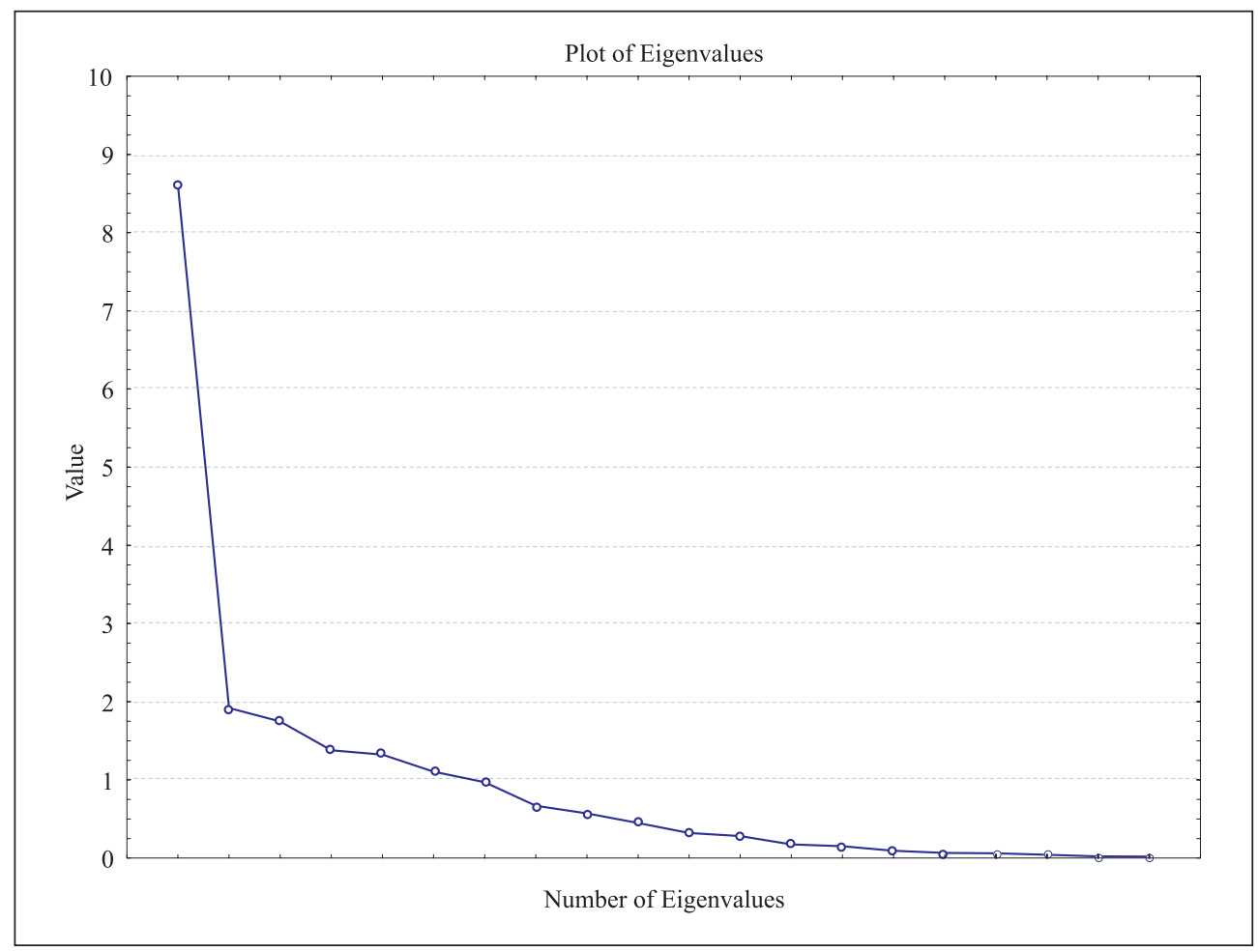

Note: The analyses were carried out by means of Statistica 8.0 and MS Excel.

Source: Authors' calculations based on European Commission, Innovation Union Scoreboard 2015, Internal Market Industry, Entrepreneurship and SMEs, Belgium 2015, Annex A: Current performance, pp. 82-83, http://ec.europa.eu/growth/industry/innovation/factsfigures/scoreboards/index_en.htm

In the following step, clustering of EU countries was done using Ward's hierarchical method with Euclidean distance and $k$-means method $^{6}$. To evaluate the variables reduction results we considered two cases: clustering based on the full list of 25 variables (Case A) and clustering based on the reduced list (Case B). Tree diagrams after hierarchical clustering for both cases are presented in Figure 5. Moreover, we compared selected four clusters of countries obtained from $k$-means method $(k=4)$ to the classification of EU countries based on SII (see Table 5 and Figure 6).

$\overline{6}$ The used $k$-means clustering aims to partition $n$ observations into $k$ clusters in which each observation belongs to the cluster with the nearest mean, serving as a prototype of the cluster. 
Figure 5: Tree diagrams for clustering EU countries by using Ward's method with Euclidean distance

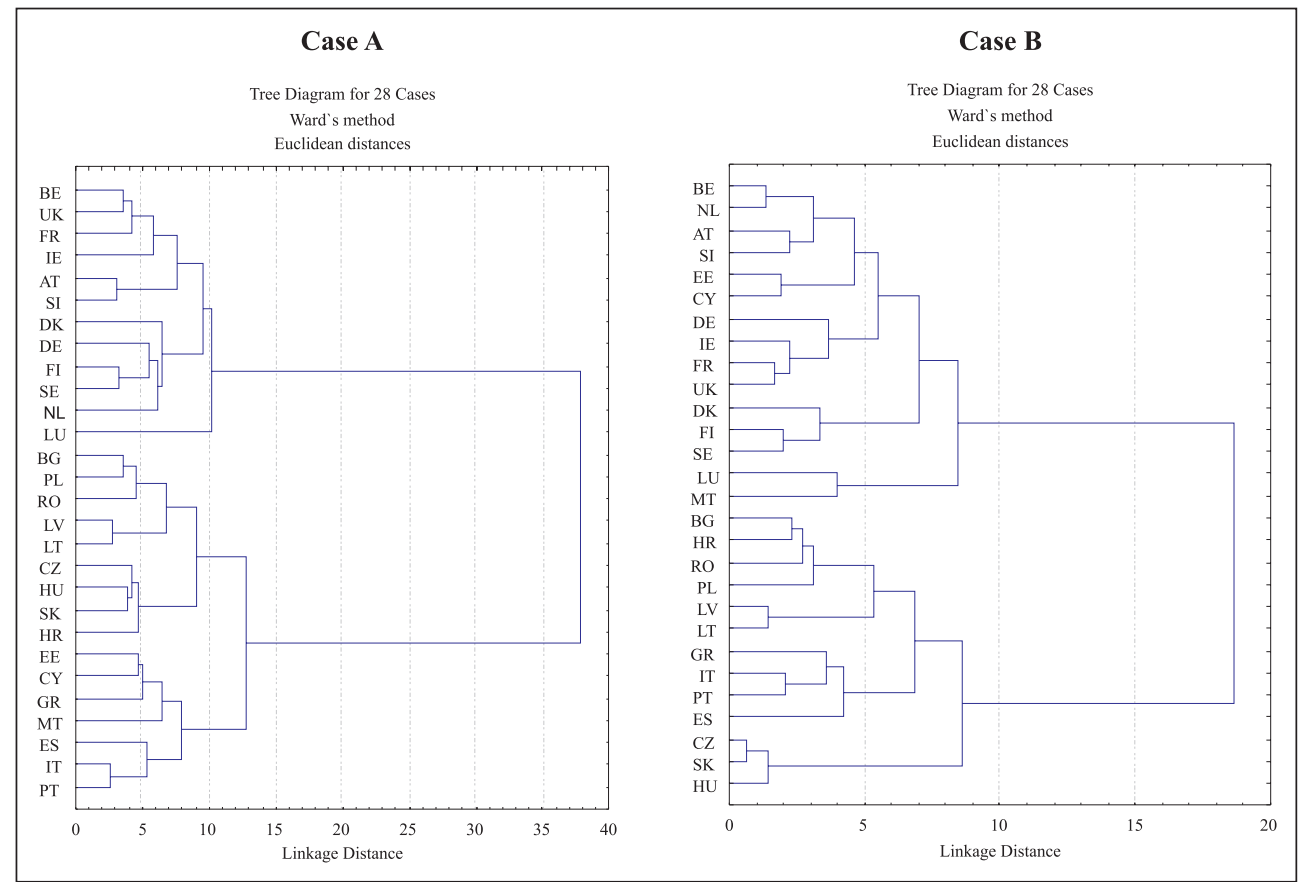

Note: The analyses were carried out by means of Statistica 8.0 and MS Excel.

Source: Authors' calculations based on European Commission, Innovation Union Scoreboard 2015, Internal Market Industry, Entrepreneurship and SMEs, Belgium 2015, Annex A: Current performance, pp. 82-83, http://ec.europa.eu/growth/industry/innovation/factsfigures/scoreboards/index_en.htm

The last stage of the analysis is establishing EU countries' rating by their innovation performance. For this reason, linear ordering method was applied, weighed and unweighed variant. Variables which serve as stimulators for innovativeness potential, were first standardised, then, two synthetic measures were created: $M_{1 k}$ (unweighed variant) and $M_{2 k}$ (weighted variant) for each country $k=1,2, \ldots, 28$, i.e.:

$$
\begin{aligned}
& M_{1 k}=\frac{1}{m} \sum_{i=1}^{m} z_{i k}, \\
& M_{2 k}=\frac{1}{\lambda} \sum_{i=1}^{m} \lambda_{i} z_{i k},
\end{aligned}
$$


where:

$z_{i k}$ - standardised value of each variable $(i)$ established for a specific country $(k)$; $m$ - the number of other analysed variables $(m=9), \lambda_{i}$ - weight related to $i-$ the variable set. The $i$ - th weight is the quotient where the numerator is an identified variance multiplied by the factor from which the variable is derived, divided by summary percentage of the variance identified by all factors, while denominator is the number of variables creating a particular factor, i.e. $\lambda=\Sigma \lambda_{i}$. The higher the synthetic factor value, the higher a given country's innovation performance is. In the case of weighted variant authors took the following weights for variables: $8.59 \%\left(X_{2}\right), 8.25 \%\left(X_{3}\right), 17.81 \%\left(X_{4}\right), 17.81 \%\left(X_{13}\right), 17.81 \%\left(X_{13}\right), 17.81 \%\left(X_{14}\right)$, $11.96 \%\left(X_{16}\right), 6.89 \%\left(X_{19}\right), 5.44 \%\left(X_{20}\right)$ and $5.44 \%\left(X_{22}\right)$.

\section{Results and discussion}

Achieved results (see table 5 and figure 6), based on self-established statistical procedure, allows to formulated following remarks. In all of the cases (see table 5) - Sweden, Denmark, Germany and Finland, are classified in the strongest group according to their innovation level. In SII classification this is the smallest group consisting of only 4 representatives, aforementioned above, while in Case $\mathrm{A}$ - this group accounts for 12 members and in Case B - 11 members. $K$-means clustering expanded the first group by Belgium, Ireland, France, Netherlands, Austria, Slovenia, and the United Kingdom. Means for cluster 1 (case A) almost for all the variables are higher than means for other clusters, with only exception for $\mathrm{X}_{10}$, which is non- $R \& D$ expenditures as $\%$ of GDP. The biggest disparities between cluster 1 and other clusters (case A) are marked for variable $\mathrm{X}_{9}$ - business $\mathrm{R} \& \mathrm{D}$ expenditures as \% of GDP and $\mathrm{X}_{15}$ - PCT patent application in societal challenges per billion GDP. The disparities are not so big between cluster 1 and the rest of the clusters when considering results in case B. There, only for 4 out of 9 variables, cluster means are the highest. The biggest inconsistency appears in cluster 4 according to SII there are only 3 representatives - Latvia, Bulgaria and Romania. To the aforementioned, the closest results are presented in case A. There, apart from 3 members, there are 2 more added, Lithuania and Poland. In case B the results are more different, the cluster is represented by Cyprus, Luxembourg and Malta. Analysis based on the cluster means proves that in case B representatives of group 4 are not the worst regarding their innovation level. The cluster mean, only for $\mathrm{X}_{3}$ - percentage youth aged 20-24 having attained at least upper secondary level education is the lowest, however mean values for all the clusters are very similar. The biggest migration of objects is noticed in clusters 2 and 3, where we have in total approximately not less than 11 representatives. In general these differences are due to the fact, that representatives of cluster 3 in SII are in case A and B divided between two clusters: second and third. At the same time, the majority of members from cluster number 2 in SII, migrates to the cluster 1 in case A and case B. 
Table 5: EU members of four clusters after $k$-means clustering $(k=4)$ and according to SII

\begin{tabular}{|c|l|l|l|}
\hline Cluster No. & $\begin{array}{c}\text { Members } \\
\text { according to SII }\end{array}$ & $\begin{array}{c}\text { Members according to } \\
k \text {-means clustering based } \\
\text { on the full list of variables }\end{array}$ & $\begin{array}{c}\text { Members according to } \\
k \text {-means clustering based on } \\
\text { the reduced list of variables }\end{array}$ \\
\hline 1 & SE, DK, DE, FI & $\begin{array}{l}\text { BE, DK, DE, IE, FR, LU, } \\
\text { NL, AT, SI, FI, SE, UK }\end{array}$ & $\begin{array}{l}\text { BE, DK, DE, IE, FR, NL, } \\
\text { AT, SI, FI, SE, UK }\end{array}$ \\
\hline 2 & $\begin{array}{l}\text { NL, LU, UK, IE, } \\
\text { BE, FR, AT, SI }\end{array}$ & $\begin{array}{l}\text { EE, GR, ES, IT, CY, MT, } \\
\text { PT }\end{array}$ & $\begin{array}{l}\text { BG, EE, GR, ES, HR, IT, } \\
\text { LV, LT, PT }\end{array}$ \\
\hline 3 & $\begin{array}{l}\text { EE, CZ, CY, IT, } \\
\text { PT, MT, ES, HU, } \\
\text { GR, SK, HR, PL, HR, HU, SK } \\
\text { LT }\end{array}$ & CZ, HU, PL, RO, SK \\
\hline 4 & LV, BG, RO & BG, LV, LT, PL, RO & CY, LU, MT \\
\hline
\end{tabular}

Note: The analyses were carried out by means of Statistica 8.0 and MS Excel.

Source: Authors' calculations based on European Commission, Innovation Union Scoreboard 2015, Internal Market Industry, Entrepreneurship and SMEs, Belgium 2015, Annex A: Current performance, pp. 82-83, http://ec.europa.eu/growth/ industry/innovation/facts-figures/scoreboards/index_en.htm

Assignment of a given country to a specified group of countries with similar innovativeness, strongly depends on the set of variables, especially when a country is not an undisputable leader of innovativeness. Undoubtedly, there are differences between tree diagrams designed for the primary and reduced set of variables (see Figure 5).

As a primary set contains variables that are strongly correlated, once these are removed, there is a change in orientation. Repetition of statistical information is being eliminated and the focus goes to different variables. In the primary set of diagnostic variables, business $R \& D$ expenditures as \% of GDP played the main role. The use of hierarchical methods allowed to detect other variables with the strongest influence on the cluster structure by comparing their means in each cluster. In this case authors could distinguish the following variables (see Figure 6): Public-private co-publications per million population, PCT patents applications or Community trademarks as well as Percentage youth aged 20-24 having attained at least upper secondary level education. The rest of diagnostic variables are also important for assessing innovation performance since they were detected in factor analysis, however their influence seems to be limited.

Tree diagrams, presented in the previous section and the last two columns in Table 5 confirm that reduction of variables influences on the cluster structure. In the case of hierarchical clustering (see Figure 5) the differences between clusters rise especially for low levels of aggregation and finally, on the biggest level of aggregation, we have 
two, almost identical clusters. In the case of $k$-means clustering with $k=4$ (see Table 5 and Figure 6) we can notice differences between clusters not only among cases A and $\mathrm{B}$ but also in comparison to clusters according to SII methodology.

Figure 6: Plot of means for clusters obtained in $k$-means clustering for cases A and B

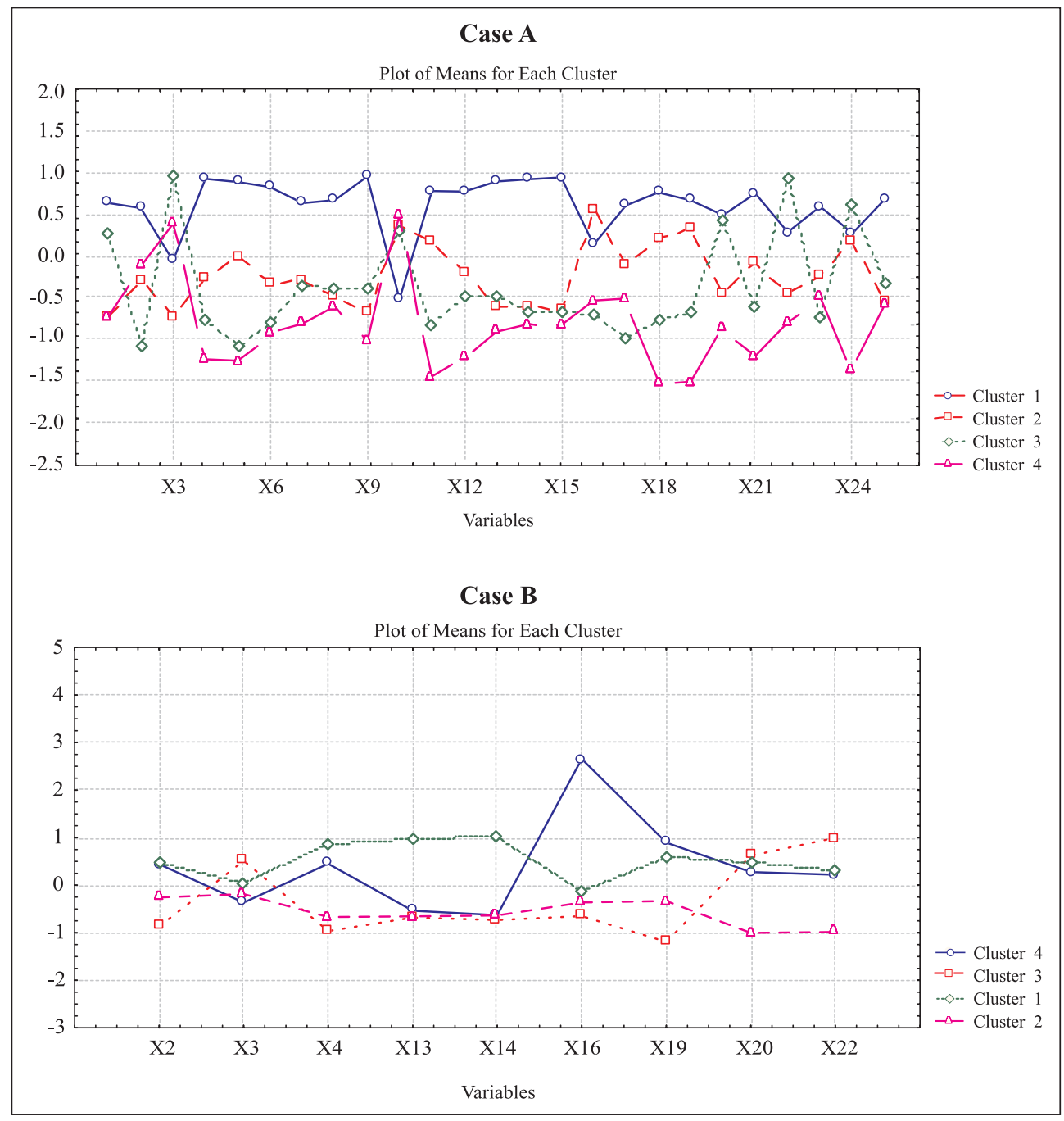

Note: The analyses were carried out by means of Statistica 8.0 and MS Excel.

Source: Authors' calculations based on European Commission, Innovation Union Scoreboard 2015, Internal Market Industry, Entrepreneurship and SMEs, Belgium 2015, Annex A: Current performance, pp. 82-83, http://ec.europa.eu/growth/ industry/innovation/facts-figures/scoreboards/index_en.htm 
Thus, a general remark can be formulated here that a classification of the given country to the specified group of countries with similar innovativeness strongly depends on the set of used variables. Moreover, even if this classification is based on the full list of considered variables it may differ strongly from the classification based on one, synthetic measure (like SII), which also takes into consideration all variables. Making a comparison of variables means in each cluster leads to detection of variables with the strongest influence on the clusters structure. In this research authors pointed out the following such variables: $X_{9}, X_{13}, X_{14}$ or $X_{16}$ (see Figure 6).

Results of comparative analysis of EU member states' ratings created by means of linear ordering and Summary Innovation Index is presented in Table 6. The higher innovation performance is confirmed by the higher synthetic factor value $-M_{1 k}$ and $M_{2 k}$. The top five in the unweighted variant are Sweden, Luxembourg, Denmark, Ireland and Finland. While in weighted variant the highest positions in the rating goes to Sweden, Denmark, Finland, Luxembourg, the Netherlands. Rating based on synthetic measure with weights shows more similarities to SII.

Table 6: EU countries rating by innovation performance assessed by $M_{1 k}, M_{2 k}$, measures, and Summary Innovation Index

\begin{tabular}{|c|c|c|c|c|c|c|}
\hline \multirow{2}{*}{ Country } & \multicolumn{2}{|c|}{$\begin{array}{c}\text { Summary Inovation } \\
\text { Index (SII) }\end{array}$} & \multicolumn{2}{c|}{$\begin{array}{c}\text { Synthetic measure } \\
\text { (without weights } \mathrm{M}_{1 \mathrm{k}} \text { ) }\end{array}$} & \multicolumn{2}{c|}{$\begin{array}{c}\text { Synthetic measure } \\
\text { (with weights } \text { M }_{\mathrm{k}} \text { ) }\end{array}$} \\
\cline { 2 - 7 } & Score & Rank & Score & Rank & Score & Rank \\
\hline SE & 0.740 & 1 & 1.041 & 1 & 1.334 & 1 \\
\hline DK & 0.736 & 2 & 0.75 & 3 & 1.194 & 2 \\
\hline FI & 0.676 & 3 & 0.573 & 5 & 0.879 & 3 \\
\hline DE & 0.676 & 4 & 0.471 & 6 & 0.45 & 8 \\
\hline NL & 0.647 & 5 & 0.348 & 9 & 0.671 & 5 \\
\hline LU & 0.642 & 6 & 0.841 & 2 & 0.801 & 4 \\
\hline UK & 0.636 & 7 & 0.345 & 10 & 0.337 & 10 \\
\hline IE & 0.628 & 8 & 0.676 & 4 & 0.454 & 7 \\
\hline BE & 0.619 & 9 & 0.23 & 13 & 0.408 & 9 \\
\hline FR & 0.591 & 10 & 0.457 & 7 & 0.286 & 12 \\
\hline AT & 0.585 & 11 & 0.442 & 8 & 0.515 & 6 \\
\hline SI & 0.534 & 12 & 0.261 & 12 & 0.303 & 11 \\
\hline EE & 0.489 & 13 & -0.188 & 18 & -0.181 & 15 \\
\hline CZ & 0.447 & 14 & -0.081 & 15 & -0.297 & 16 \\
\hline CY & 0.445 & 15 & 0.261 & 11 & 0.196 & 13 \\
\hline IT & 0.439 & 16 & -0.371 & 20 & -0.407 & 18 \\
\hline PT & 0.403 & 17 & -0.654 & 25 & -0.604 & 24 \\
\hline MT & 0.397 & 18 & 0.033 & 14 & -0.139 & 14 \\
\hline & & & & & & \\
\hline
\end{tabular}




\begin{tabular}{|c|c|c|c|c|c|c|}
\hline \multirow{2}{*}{ Country } & \multicolumn{2}{|c|}{$\begin{array}{c}\text { Summary Inovation } \\
\text { Index (SII) }\end{array}$} & \multicolumn{2}{c|}{$\begin{array}{c}\text { Synthetic measure } \\
\text { (without weights } \mathrm{M}_{\mathrm{lk}} \text { ) }\end{array}$} & \multicolumn{2}{c|}{$\begin{array}{c}\text { Synthetic measure } \\
\text { (with weights } \text { 2 }_{\mathrm{k}} \text { ) }\end{array}$} \\
\cline { 2 - 7 } & Score & Rank & Score & Rank & Score & Rank \\
\hline ES & 0.385 & 19 & -0.588 & 24 & -0.521 & 21 \\
\hline HU & 0.369 & 20 & -0.146 & 16 & -0.362 & 17 \\
\hline GR & 0.365 & 21 & -0.459 & 21 & -0.499 & 20 \\
\hline SK & 0.360 & 22 & -0.182 & 17 & -0.448 & 19 \\
\hline HR & 0.313 & 23 & -0.512 & 22 & -0.551 & 22 \\
\hline PL & 0.313 & 24 & -0.367 & 19 & -0.581 & 23 \\
\hline LT & 0.283 & 25 & -0.584 & 23 & -0.605 & 25 \\
\hline LV & 0.272 & 26 & -0.884 & 28 & -0.834 & 26 \\
\hline BG & 0.229 & 27 & -0.848 & 26 & -0.849 & 27 \\
\hline RO & 0.204 & 28 & -0.867 & 27 & -0.952 & 28 \\
\hline
\end{tabular}

Note: The analyses were carried out by means of Statistica 8.0 and MS Excel.

Source: Authors' calculations based on European Commission, Innovation Union Scoreboard 2015, Internal Market Industry, Entrepreneurship and SMEs, Belgium 2015, Annex A: Current performance, pp. 82-83, http://ec.europa.eu/growth/ industry/innovation/facts-figures/scoreboards/index_en.htm

Authors obtained high rank correlation coefficients established for comparative ratings (Table 7 and Table 8), although central positions in the ratings reveal major differences (Table 6).

Table 7: Comparison EU countries ratings by means of Spearman correlation coefficient

\begin{tabular}{|l|c|c|c|}
\hline \multicolumn{1}{|c|}{ Spearman correlations } & $M_{1 k}$ & $M_{2 k}$ & $S I I$ \\
\hline$M_{1 k}$ & 1.00 & 0.95 & 0.91 \\
\hline$M_{2 k}$ & 0.95 & 1.00 & 0.96 \\
\hline SII & 0.91 & 0.96 & 1.00 \\
\hline
\end{tabular}

Note: The analyses were carried out by means of Statistica 8.0 and MS Excel.

Source: Authors' calculations based on European Commission, Innovation Union Scoreboard 2015, Internal Market Industry, Entrepreneurship and SMEs, Belgium 2015, Annex A: Current performance, pp. 82-83, http://ec.europa.eu/growth/ industry/innovation/facts-figures/scoreboards/index_en.htm

High correlations are justified, due to the fact, that positions of the most innovative countries and these with the lowest innovativeness performance are not threatened regardless of the set of diagnostic variables - primary or reduced. 
Table 8: Comparison EU countries ratings by means of Kendall correlation coefficient

\begin{tabular}{|l|c|c|c|}
\hline \multicolumn{1}{|c|}{ Kendall correlations } & $M_{1 k}$ & $M_{2 k}$ & SII \\
\hline$M_{1 k}$ & 1.00 & 0.85 & 0.76 \\
\hline$M_{2 k}$ & 0.85 & 1.00 & 0.85 \\
\hline SII & 0.76 & 0.85 & 1.00 \\
\hline
\end{tabular}

Note: The analyses were carried out by means of Statistica 8.0 and MS Excel.

Source: Authors' calculations based on European Commission, Innovation Union Scoreboard 2015, Internal Market Industry, Entrepreneurship and SMEs, Belgium 2015, Annex A: Current performance, pp. 82-83, http://ec.europa.eu/growth/ industry/innovation/facts-figures/scoreboards/index_en.htm

The results obtained work here as a justification of a statistical procedure discussed in the paper, mainly, due to the fact that linear ordering for the EU member countries conducted by authors is convergent with the rating based on SII. Authors noticed that limiting to variables with the biggest values of factor loadings (i.e. $X_{4}$, $X_{13}, X_{14}$ - variables included in factor no. 1 as well as others - see Table 3) leads to crucial changes in evaluation of most of EU countries innovativeness. Moreover, the reduction process has an influence on the clusters' structure (see Table 5) and thus it should not be omitted. The minimalist tendency is also characteristic in application of multidimensional statistics (Jenkins and Andersen, 2003).

\section{Conclusions}

The results of the conducted research confirmed the earlier stated hypothesis that the initial number of diagnostic variables describing the innovativeness of EU member states can be reduced effectively by the use of multidimensional statistics. The obtained results are the contribution to the economic literature by: (1) empirical study on distinguishing the most important indicators of innovativeness, (2) selfelaborated proposal of a new synthetic measure of innovation performance based on a reduced set of diagnostic variables, and finally - (3) creation of EU member states rating according to the aforementioned synthetic index. This paper is also an attempt to provide scientific contribution to the reconciliation of the measurement of innovativeness by focusing on selected and the most important innovativeness detectors. These results can be translated into economic and social works on construction of innovativeness models. Moreover, the procedure of selecting diagnostic variables and weighting system calls for special particularization. It is confirmed by the proposal of two different linear orderings presented in weighted and unweighted variant. Giving a privilege to the first one, it attempts to reaffirm the one way of thinking, presented in the economic literature, which regards all 
the innovation determinants equally. Obviously, an individual proposal based on statistical approach may meet with many limitations and problems, like data accessibility or data topicality. The limit of the research is given by variables used for creation of Summary Innovation Index itself, and is expressed by the fact that data comes from different periods of time. The self-developed synthetic measure is directly affected by this problematic issue as SII is considered as a starting point in creation of it. It is important to understand that innovativeness synthetic measures usually contain simultaneously input and output indicators. Moreover, in economics it is not an unusual issue to have a delay between an economic action and a consequence of it. An impact of time lags is of a crucial importance here. However, this problem was minimized, at least to some extent, by using the reduced set of variables for the final linear ordering and eliminating the oldest data available. It is important to continue this research by focusing on analyzing the dynamics of self-developed synthetic index on longer periods of time. It will enable assessment of changes in time of innovation performance in the EU based on reduced set of variables and also comparative analysis with SII. The present research should be extended by the use of other multidimensional statistical methods and selected data mining methods for detecting indicators that impact innovation performance to the most. Moreover, the future of this research is to concentrate on seeking relations between all detected indicators and forecasting (by using econometric models) the innovativeness of EU member states. The obtained results should be the basis for further development of the measurement of innovativeness. Researchers are requested to put a greater emphasis in this specific topic on: a) the use of multivariate statistical methods for the final selection of variables; b) in depth analysis of the limited number of variables to get a clear picture of innovativeness' main driving forces and for better understanding of the current innovation performance on the country level. This analysis is also important for developing suitable international economic policies in order to achieve a better innovation performance in the EU. The existence of certain clusters identified with k-means method might be helpful for the EU policy-makers. They should pay attention to the fact, that the best solution for moderate innovators might be to follow very narrow country specific recommendations, rather than more general international standards. Usefulness of this research also should be reflected in economic goals setting on a country level. In particular, politicians should follow key points like: (1) support to businesses investing in R\&D, and (2) provision of favourable conditions for scientists interested in cooperation with the private sector.

\section{References}

Allen, J.A. (1966) Scientific innovation and industrial prosperity, London: Longman.

Bielski, I. (2000) The course and determinants of innovation processes, Bydgoszcz: OPO. 
Cattell, R. B. (1966) "The screen test for the number of factors", Multivariate Behavioral Research, Vol. 1, No. 2, pp. 245-276, doi: 10.1207/ s15327906mbr0102_10.

Carter, C.F., Williams, B.R. (1958) Industry and technological process, London: Oxford University Press.

Child, D. (2006) The Essentials of Factor Analysis (3rd ed.), London \& New York: Continuum, International Publishing Group.

Coe, D., Helpman, E. (1995) "International R\&D spillovers", European Economic Review, Vol. 39, No. 5, pp. 859-887, doi: 10.1016/0014-2921(94)00100-e.

Dąbrowa M. (2014) "Is Polish economy innovative?", Science papers of Malopolska Economic University, Vol. 24, No 1, p. 74.

David, P., Hall, B., Toole, A. (2000) "Is public R\&D a complement or substitute for private R\&D? A review of the econometric evidence", Research Policy, Vol. 29, No. 4-5, pp. 497-529, doi: 10.1016/S0048-7333(99)00087-6.

Domar, E. (1946) "Capital expansion, rate of growth, and employment", Econometrica, Vol. 14, No. 2, pp. 137-147, doi: 10.2307/1905364.

European Commission (2010) Europe 2020 Flagship Initiative Innovation Union, Communication From The Commission To The European Parliament, The Council, The European Economic And Social Committee And The Committee Of The Regions, SEC(2010) 1161, Brussels, 6.10.2010, COM(2010) 546 final, http:// ec.europa.eu/research/innovation-union/pdf/innovation-union-communication_ en.pdf.

European Commission (2015) Innovation Union Scoreboard 2015, Internal Market Industry, Entrepreneurship and SMEs, Belgium.

Everitt, B., Landau, S., Leese, M., Stahl, D. (2011) Cluster analysis, Chichester, West Sussex, U.K: Wiley, doi: 10.1002/9780470977811.

Fagerberg, J. (1987) "A Technology Gap Approach to Why Growth Rates Differ", Research Policy, Vol. 16, No. 4, pp. 87-99, doi: 10.1016/0048-7333(87)90025-4.

Freeman,Ch. (1994) "The economics of technical change", Cambridge Journal of Economics, Vol. 18, No. 5, pp. 463-514.

Goldsmith, R.E., Foxall, G.R. (2003) "The Measurement of Innovativeness", In Shavinina, L.V. (ed.), Prague: MAC Prague consulting Ltd, doi: 10.1016/b978008044198-6/50022-x.

Haffer, M. (1998) Determinanty strategii nowego produktu polskich przedsiębiorstw przemystowych, Toruń: UMK.

Harrod, R.F. (1939) “An essay in dynamic theory”, Economic Journal, Vol. 49, No. 193, pp. 14-33, doi: 10.2307/2225181.

Heilbroner, R. R. (1993) Large-scale economists. Times. Life Ideas, Warsaw: PWE. Hollanders, H., Van Cruysen, A. (2008) Rethinking the European Innovation Scoreboard: A New Methodology for 2008-2010, Genewa: Pro INNO Europe. 
Hollanders, H., Tarantola, S. (2011) Innovation Union Scoreboard 2010 Methodology report, Genewa: Pro INNO Europe.

Janasz, W., Kozioł, K. (2007) Determinants of innovation processes in firms, Warsaw: PWE.

Jenkins, L., Andersen, M. (2003) "A multivariate statistical approach to reducing the number of variables in data envelopment analysis", European Journal of Operational Research, Vol. 147, No. 1, pp. 51-61, doi: 10.1016/s03772217(02)00243-6.

Kaiser, H.F. (1960) "The application of electronic computers to factor analysis", Educational and Psychological Measurement, Vol. 20, No. 1, pp. 141-151, doi: 10.1177/001316446002000116.

Martínez-Pellitero, M., Buesa, M., Heijs, J. Y., Baumert, T. (2008) “A Novel Way of Measuring Regional Systems of Innovation: Factor Analysis As a Methodological Approach", Madrid: IAIF Working Paper - Documento De Trabajo No. 60.

Munsfield, E. (1968) Industrial Research and Technology Innovation, New York: Norton W.W. and Co.

Niedbalska, G., (2011) Stownik Innowacji, http://www.pi.gov.pl/PARP/.

Niedzielski, P., Rychlik, K. (2006), Innovation and creativity, Szczecin: University of Szczecin Press.

North, D. C. (1981) Structure and Change in Economic History, New York: W. W. Norton, doi: 10.1177/003232928201100416.

North, D. C. (2005) Understanding the process of Economic Change, Princeton: Princeton University Press, doi: 10.1515/9781400829484.

Piech, K., (2009) Wiedza i innowacje $w$ rozwoju gospodarczym: $w$ kierunku pomiaru i wspótczesnej roli państwa, Warszawa: Instytut Wiedzy i Innowacji.

Porter, M.E. (1990) The competitive Advantage of Nations, London: The Macmillan Press, doi: 10.1007/978-1-349-11336-1.

Porter M.E. (2001) Clusters of Innovation: Regional Foundations of U.S. Competivness, Washington: Council of Competitiveness.

Radicic, D., Pugh, G., Hollanders, H., Wintjes, R. (2014) The impact of innovation support pro-grammes on SME innovation in traditional manufacturing industries: an evaluation for seven EU regions, Brussels: UNU-MERIT Working Paper Series, doi: 10.2139/ssrn.2438459.

Rogers, E.M. (2003) Diffusion of innovations, New York: Free Press.

Romer, P. (1986) "Increasing returns and long-run growth", Journal of Political Economy, Vol. 94, No. 5, pp. 1002-1037, doi: 10.1086/261420.

Saixing, Z. , Xuemei, X., Chiming, T. (2010) "Evaluating innovation capabilities for science parks: A system model", Ukio Technologinis ir Ekonominis Vystymas, Vol. 16, No. 3, pp. 397-413, doi: 10.3846/tede.2010.25. 
Schibany, A. (2008) How not to compare innovation performance. A critical assessment of the European Innovation Scoreboard, Institute of Technology and Regional Policy - InTeReg.

Schumpeter, J.A. (1942) Capitalism, socialism and democracy, New York: Harper \& Row.

Schumpeter, J.A. (1960) Economic growth theory, Warsaw: PWN.

Solow, R. M. (1956) "A contribution to the theory of economic growth", Quarterly Journal of Economics, Vol. 70, No. 1, pp. 65-94, doi: 10.2307/1884513.

Solow, R. M. (1957) "Technical change and the aggregate production function", Review of Economics and Statistics, Vol. 39, No. 3, pp. 312-320, doi: 10.2307/1926047.

Swan, T.W. (1956) "Economic growth and capital accumulation", Economic Record, Vol. 32, No. 2, pp. 334-361, doi: 10.1111/j.1475-4932.1956.tb00434.x.

Thompson, B. (2004) Exploratory and confirmatory factor analysis: Understanding concepts and applications, Washington DC: American Psychological Association, doi: 10.1037/10694-000.

Uppenberg, K. (2009) "Innovation and economic growth", EIB Papers, Vol. 14, No. 1, pp. 11-35, doi: 10.2139/ssrn.1828904.

Ward, J. H. (1963) "Hierarchical Grouping to Optimize an Objective Function", Journal of the American Statistical Association, Vol. 58, No. 301, pp. 236-244, doi: 10.2307/2282967.

Walesiak, M. (1996) Metody analizy danych marketingowych, Warsaw: PWN.

Whitfield, P.R. (1979) Innowacje w przemyśle, Warsaw: PWE.

Veblen, T. (1971) The Theory of the Leisure Class, Warsaw: PWN.

Yuan-Chieh, Ch., Ming-Huei, Ch., Yuan-Po, L., et al. (2012) "Measuring Regional Innovation and Entrepreneurship Capabilities. The Case of Taiwan Science Parks", Journal of the Knowledge Economy, Vol. 3, No. 2, pp. 90-108, doi: 10.1007/s13132-011-0081-4. 


\title{
Multivarijantni pristup u mjerenju inovacija
}

\author{
Elżbieta Roszko-Wójtowicz ${ }^{1}$, Jacek Białek ${ }^{2}$
}

\begin{abstract}
Sažetak
Cilj ovog istraživanja je predložiti postupak mjerenja inovativnosti primjenjujući metodologiju Sažetka indeksa inovacija (Summary Innovation Index methodology - SII) kao početnu točku. U suvremenom svijetu, inovativna aktivnost percipira se kao resurs konkurentnosti i gospodarskog rasta. Novi proizvodi, modeli korisnosti, zaštitni znakovi i kreativni projekti važni su elementi sadašnje društveno-ekonomske stvarnosti. Konkretno, autori su usredotočeni na izbor i primjenu multivarijantne statističke analize u svrhu razlučivanja čimbenika koji najviše utječu na inovativnost gospodarstava EU. Rezultat kvantitativne analize je linearni poredak zemalja EU prema razini njihove inovativnosti na temelju reduciranog skupa dijagnostičkih varijabli. Ocjenjivanje se usporedilo $s$ rezultatima prikazanim $u$ Sažetku indeksa inovacija. Provedena analiza pokazuje konvergenciju između autorovih rezultata i postojećih ocjena inovativnosti. Ipak, glavni zaključak je da metodologija procjene inovativnosti ostaje otvoreno pitanje i zahtijeva daljnja istraživanja. Prije svega, potrebno se osobito koncentrirati na detaljniju provjeru malog skupa varijabli koje imaju najveći utjecaj na inovativnost. Ujedno, obostrani je interes gospodarstva i društva da se dobije jasna slika o pokretačkoj snazi inovativnosti.
\end{abstract}

Ključne riječi: inovativnost, Unija inovacija, Europska unija, klaster analiza, faktorska analiza, teorija indeksa cijena

JEL klasifikacija: C380, O300

1 Docentica, Department of Economic and Social Statistics, Faculty of Economics and Sociology, University of Lodz, Rewolucji 1905 r. No. 41, 90-214 Lódź, Poljska. Znanstveni interes: obiteljsko poduzetništvo/poduzeća, cijeloživotno učenje, inovativnost, javna nabava, statistika u ekonomskim i društvenim istraživanjima. Tel.: +48 42635 4831. E-mail: eroszko33@gmail. com. Osobna web stranica: http://www.kseis.uni.lodz.pl/pracownicy/pracownik/id/13.

2 Docent s habilitacijom, Department of Statistical Methods, Faculty of Economics and Sociology, University of Lodz, Rewolucji 1905 r. No. 41/43, 90-214 Łódź, Poljska. Znanstveni interes: matematička statistika, teorija indeksa cijena, otvoreni mirovinski fondovi. Tel.: +4842635 5182. E-mail: jbialek@uni.lodz.pl..Osobna web stranica: http://www.statystyka.uni.lodz.pl/ pracownicy/dr-hab-inz-jacek-bialek/. 
Elżbieta Roszko-Wójtowicz, Jacek Białek • A multivariate approach in measuring...

Zb. rad. Ekon. fak. Rij. • 2016 • vol. $34 \cdot$ no. $2 \cdot 443-479$

\section{Appendix}



Elżbieta Roszko-Wójtowicz, Jacek Białek • A multivariate approach in measuring...

Table A1: Changes in Innovation Union Scoreboard methodology

\begin{tabular}{|c|c|c|}
\hline $\begin{array}{c}\text { European Innovation Scoreboard (EIS) } \\
2009 \\
\end{array}$ & $\begin{array}{c}\text { Innovation Union } \\
\text { Scoreboard (IUS) } 2010 \\
\end{array}$ & Changes / Adjustments \\
\hline ENABLERS & ENABLERS & \\
\hline Human resources & Human resources & \\
\hline $\begin{array}{l}\text { 1.1.1 S\&E and SSH graduates }\left(1^{\text {st }}\right. \\
\text { stage) per } 1000 \text { population aged } 20-29\end{array}$ & $\mathrm{n} / \mathrm{a}$ & $\begin{array}{l}\text { EIS } 2009 \text { indicator no longer } \\
\text { used }\end{array}$ \\
\hline $\begin{array}{l}\text { 1.1.2 S\&E and SSH doctorate } \\
\text { graduates }\left(2^{\text {nd }} \text { stage }\right) \text { per } 1000 \\
\text { population aged } 25-34\end{array}$ & $\begin{array}{l}\text { 1.1.1 New doctorate } \\
\text { graduates (ISCED 6) per } \\
1000 \text { population aged 25-34 } \\
\end{array}$ & $\begin{array}{l}\text { Broader definition than that } \\
\text { used in the EIS } 2009\end{array}$ \\
\hline $\begin{array}{l}\text { 1.1.3 Population with tertiary } \\
\text { education per } 100 \text { population aged } \\
25-64\end{array}$ & $\begin{array}{l}\text { 1.1.2 Percentage population } \\
\text { aged } 30-34 \text { having } \\
\text { completed tertiary education }\end{array}$ & $\begin{array}{l}\text { Age group more narrowly } \\
\text { defined than in EIS } 2009\end{array}$ \\
\hline $\begin{array}{l}\text { 1.1.4 Participation in life-long learning } \\
\text { per } 100 \text { population aged } 25-64\end{array}$ & $\mathrm{n} / \mathrm{a}$ & $\begin{array}{l}\text { EIS } 2009 \text { indicator no longer } \\
\text { used }\end{array}$ \\
\hline 1.1.5 Youth education attainment level & $\begin{array}{l}\text { 1.1.3 Percentage youth aged } \\
\text { 20-24 having attained at } \\
\text { least upper secondary level } \\
\text { education }\end{array}$ & $\begin{array}{l}\text { Different names but identical } \\
\text { indicators }\end{array}$ \\
\hline $\mathrm{n} / \mathrm{a}$ & $\begin{array}{l}\text { Open, excellent and } \\
\text { attractive research systems }\end{array}$ & \\
\hline $\mathrm{n} / \mathrm{a}$ & $\begin{array}{l}\text { 1.2.1 International scientific } \\
\text { co-publications per million } \\
\text { population }\end{array}$ & New indicator \\
\hline $\mathrm{n} / \mathrm{a}$ & $\begin{array}{l}\text { 1.2.2 Scientific publications } \\
\text { among the top } 10 \% \text { most } \\
\text { cited publications worldwide } \\
\text { as \% of total scientific } \\
\text { publications of the country }\end{array}$ & New indicator \\
\hline $\mathrm{n} / \mathrm{a}$ & $\begin{array}{l}1.2 .3 \text { Non-EU doctorate } \\
\text { students as \% of all } \\
\text { doctorate students } \\
\end{array}$ & New indicator \\
\hline Finance and support & Finance and support & \\
\hline $\begin{array}{l}\text { 1.2.1 Public R\&D expenditures as \% } \\
\text { of GDP }\end{array}$ & $\begin{array}{l}\text { 1.3.1 Public R\&D } \\
\text { expenditures as \% of GDP }\end{array}$ & Identical \\
\hline 1.2.2 Venture capital as $\%$ of GDP & $\begin{array}{l}\text { 1.3.2 Venture capital (early } \\
\text { stage, expansion and } \\
\text { replacement) as \% of GDP }\end{array}$ & $\begin{array}{l}\text { Different names but identical } \\
\text { indicators }\end{array}$ \\
\hline 1.2.3 Private credit as a $\%$ of GDP & $\mathrm{n} / \mathrm{a}$ & $\begin{array}{l}\text { EIS } 2009 \text { indicator no longer } \\
\text { used }\end{array}$ \\
\hline 1.2.4 Broadband access by firms & $\mathrm{n} / \mathrm{a}$ & \begin{tabular}{|l} 
EIS 2009 indicator no longer \\
used
\end{tabular} \\
\hline FIRM ACTIVITIES & FIRM ACTIVITIES & \\
\hline Firm investments & Firm investments & \\
\hline $\begin{array}{l}\text { 2.1.1 Business R\&D expenditures as } \\
\% \text { of GDP }\end{array}$ & $\begin{array}{l}\text { 2.1.1 Business R\&D } \\
\text { expenditures as \% of GDP }\end{array}$ & Identical \\
\hline
\end{tabular}




\begin{tabular}{|c|c|c|}
\hline $\begin{array}{c}\text { European Innovation Scoreboard (EIS) } \\
2009\end{array}$ & $\begin{array}{c}\text { Innovation Union } \\
\text { Scoreboard (IUS) } 2010\end{array}$ & Changes / Adjustments \\
\hline 2.1.2 IT expenditures as a $\%$ of GDP & $\mathrm{n} / \mathrm{a}$ & $\begin{array}{l}\text { EIS } 2009 \text { indicator no longer } \\
\text { used }\end{array}$ \\
\hline \multirow[t]{2}{*}{$\begin{array}{l}\text { 2.1.3 Non-R\&D innovation } \\
\text { expenditures as } \% \text { of turnover }\end{array}$} & $\begin{array}{l}\text { 2.1.2 Non-R\&D innovation } \\
\text { expenditures as \% of } \\
\text { turnover }\end{array}$ & Identical \\
\hline & Linkages \& entrepreneurship & \\
\hline $\begin{array}{l}\text { 2.2.1 SMEs innovating in-house as \% } \\
\text { of SMEs }\end{array}$ & $\begin{array}{l}\text { 2.2.1 SMEs innovating } \\
\text { in-house as } \% \text { of SMEs }\end{array}$ & Identical \\
\hline $\begin{array}{l}\text { 2.2.2 Innovative SMEs collaborating } \\
\text { with others as } \% \text { of SMEs }\end{array}$ & $\begin{array}{l}\text { 2.2.2 Innovative SMEs } \\
\text { collaborating with others as } \\
\% \text { of SMEs }\end{array}$ & Identical \\
\hline $\begin{array}{l}\text { 2.2.3 Firm renewal rate (SMEs entries } \\
\text { and exits as a } \% \text { of all SMEs) }\end{array}$ & $\mathrm{n} / \mathrm{a}$ & $\begin{array}{l}\text { EIS } 2009 \text { indicator no longer } \\
\text { used }\end{array}$ \\
\hline $\begin{array}{l}\text { 2.2.4 Public-private co-publications } \\
\text { per million population }\end{array}$ & $\begin{array}{l}\text { 2.2.3 Public-private } \\
\text { co-publications per million } \\
\text { population }\end{array}$ & Identical \\
\hline Throughputs & Intellectual Assets & \\
\hline $\begin{array}{l}\text { 2.3.1 EPO patent applications million } \\
\text { population }\end{array}$ & $\mathrm{n} / \mathrm{a}$ & $\begin{array}{l}\text { EIS } 2009 \text { indicator no longer } \\
\text { used }\end{array}$ \\
\hline $\mathrm{n} / \mathrm{a}$ & \begin{tabular}{|l|} 
2.3.1 PCT patent \\
applications per billion GDP \\
(in PPS€)
\end{tabular} & New indicator \\
\hline $\mathrm{n} / \mathrm{a}$ & $\begin{array}{l}\text { 2.3.2 PCT patent } \\
\text { applications in societal } \\
\text { challenges per billion GDP } \\
\text { (in PPS€) (climate change } \\
\text { mitigation; health) }\end{array}$ & New indicator \\
\hline $\begin{array}{l}\text { 2.3.2 Community trademarks per } \\
\text { million population }\end{array}$ & $\begin{array}{l}\text { 2.3.3 Community } \\
\text { trademarks per billion GDP } \\
\text { (in PPS€) }\end{array}$ & Different denominator \\
\hline $\begin{array}{l}\text { 2.3.3 Community designs per million } \\
\text { population }\end{array}$ & $\begin{array}{l}\text { 2.3.4 Community designs } \\
\text { per billion GDP (in PPS } € \text { ) }\end{array}$ & Different denominator \\
\hline $\begin{array}{l}\text { 2.3.4 Technology Balance of Payments } \\
\text { flows as \% of GDP }\end{array}$ & $\mathrm{n} / \mathrm{a}$ & $\begin{array}{l}\text { Receipts captured in IUS } \\
2010 \text { indicator } 3.2 .5\end{array}$ \\
\hline OUTPUTS & OUTPUTS & \\
\hline Innovators & Innovators & \\
\hline $\begin{array}{l}\text { 3.1.1SMEs introducing product or } \\
\text { process innovations as } \% \text { of SMEs }\end{array}$ & $\begin{array}{l}\text { 3.1.1 SMEs introducing } \\
\text { product or process } \\
\text { innovations as } \% \text { of SMEs }\end{array}$ & Identical \\
\hline $\begin{array}{l}3.1 .2 \text { SMEs introducing marketing } \\
\text { or organisational innovations as } \% \text { of } \\
\text { SMEs }\end{array}$ & $\begin{array}{l}\text { 3.1.2 SMEs introducing } \\
\text { marketing or organisational } \\
\text { innovations as \% of SMEs }\end{array}$ & Identical \\
\hline $\begin{array}{l}3.1 .3 \text { Resource efficiency innovators } \\
\text { as \% of all firms }\end{array}$ & $\mathrm{n} / \mathrm{a}$ & $\begin{array}{l}\text { EIS } 2009 \text { indicator no longer } \\
\text { used }\end{array}$ \\
\hline $\mathrm{n} / \mathrm{a}$ & \begin{tabular}{|l|} 
3.1.3 High-growth \\
innovative enterprises
\end{tabular} & New indicator \\
\hline
\end{tabular}


Elżbieta Roszko-Wójtowicz, Jacek Białek • A multivariate approach in measuring...

\begin{tabular}{|c|c|c|}
\hline $\begin{array}{c}\text { European Innovation Scoreboard (EIS) } \\
2009\end{array}$ & $\begin{array}{c}\text { Innovation Union } \\
\text { Scoreboard (IUS) } 2010\end{array}$ & Changes / Adjustments \\
\hline Economic effects & Economic effects & \\
\hline $\begin{array}{l}\text { 3.2.1 Employment in medium-high } \\
\& \text { high-tech manufacturing as } \% \text { of } \\
\text { workforce }\end{array}$ & $\mathrm{n} / \mathrm{a}$ & $\begin{array}{l}\text { EIS } 2009 \text { indicator no longer } \\
\text { used }\end{array}$ \\
\hline $\begin{array}{l}\text { 3.2.2 Employment in knowledge- } \\
\text { intensive services as \% of workforce }\end{array}$ & $\mathrm{n} / \mathrm{a}$ & $\begin{array}{l}\text { EIS } 2009 \text { indicator no longer } \\
\text { used }\end{array}$ \\
\hline $\mathrm{n} / \mathrm{a}$ & $\begin{array}{l}\text { 3.2.1 Employment in } \\
\text { knowledge-intensive } \\
\text { activities (manufacturing and } \\
\text { services) as \% of workforce }\end{array}$ & New indicator \\
\hline $\begin{array}{l}\text { 3.2.3 Medium and high-tech product } \\
\text { exports as } \% \text { of total product exports }\end{array}$ & $\begin{array}{l}\text { 3.2.2 Medium and high-tech } \\
\text { product exports as } \% \text { of total } \\
\text { product exports }\end{array}$ & Identical \\
\hline $\begin{array}{l}\text { 3.2.4 Knowledge-intensive services } \\
\text { exports as } \% \text { of total services exports }\end{array}$ & $\begin{array}{l}\text { 3.2.3 Knowledge-intensive } \\
\text { services exports as \% of } \\
\text { total services exports }\end{array}$ & Identical \\
\hline $\begin{array}{l}\text { 3.2.5 Sales of new to market } \\
\text { innovations as \% of turnover }\end{array}$ & \multirow{2}{*}{$\begin{array}{l}\text { 3.2.4 Sales of new to market } \\
\text { and new to firm innovations } \\
\text { as \% of turnover }\end{array}$} & \multirow[t]{2}{*}{$\begin{array}{l}\text { Combines EIS } 2009 \\
\text { indicators 3.2.5 and 3.2.6 }\end{array}$} \\
\hline $\begin{array}{l}\text { 3.2.6 Sales of new to firm innovations } \\
\text { as \% of turnover }\end{array}$ & & \\
\hline $\mathrm{n} / \mathrm{a}$ & $\begin{array}{l}3.2 .5 \text { Licence and patent } \\
\text { revenues from abroad as \% } \\
\text { of GDP }\end{array}$ & $\begin{array}{l}\text { Part of EIS indicator } 2.3 .4 \\
\text { on TBP flows }\end{array}$ \\
\hline
\end{tabular}

Source: Authors' self-study based on European Commission, Innovation Union Scoreboard 2015, Internal Market Industry, Entrepreneurship and SMEs, Belgium 2015 